\title{
Spectroscopy and photometry of the emission-line B-type stars AS 78 and MWC 657^
}

\author{
A.S. Miroshnichenko ${ }^{1,2}$, E.L. Chentsov ${ }^{3,4}$, V.G. Klochkova ${ }^{3,4}$, K.S. Kuratov ${ }^{5}$, T.A. Sheikina ${ }^{5}$, D.B. Mukanov ${ }^{6}$, \\ K.S. Bjorkman ${ }^{1}$, R.O. Gray ${ }^{7}$, R.J. Rudy ${ }^{8}$, D.K. Lynch $^{8}$, S. Mazuk ${ }^{8}$, R. Puetter ${ }^{9}$, P. García-Lario ${ }^{10}$, J.V. Perea ${ }^{11}$, \\ and Yu.K. Bergner ${ }^{2}$ \\ 1 Ritter Observatory, Dept. of Physics \& Astronomy, University of Toledo, Toledo, OH 43606, U.S.A. \\ 2 Central Astronomical Observatory of the Russian Academy of Sciences at Pulkovo, 196140, Saint-Petersburg, Russia \\ 3 Special Astrophysical Observatory of the Russian Academy of Sciences, Karachai-Cirkassian Republic, Nizhnij Arkhyz, \\ 357147, Russia \\ 4 Isaak Newton Institute of Chile, SAO branch, Chile \\ ${ }^{5}$ Fesenkov Astrophysical Institute of the National Academy of Sciences, Kamenskoe plato, Almaty, 480068, Kazakhstan \\ 6 Al-Farabi Kazakh National State University, Almaty, 480000, Kazakhstan \\ 7 Dept. of Physics and Astronomy, Appalachian State University, Boone, NC 28608, U.S.A. \\ 8 Aerospace Corp. M2/266, P.O. Box 92957, Los Angeles, CA 90009, U.S.A. \\ 9 Center for Astrophysics and Space Sciences, University of California, San Diego, C-0111, La Jolla, CA 92093, U.S.A. \\ 10 ISO Data Centre, Astrophysics Division, Space Science Department of ESA, Villafranca del Castillo, Apartado de Correos \\ 50727, E-28080 Madrid, Spain \\ 11 INSA S.A., Villafranca del Castillo, Apartado de Correos 50727, E-28080 Madrid, Spain
}

Received March 20; accepted July 3, 2000

\begin{abstract}
We present the results of low- and highresolution spectroscopic and multicolour photometric observations of two early-type emission-line stars, AS 78 and MWC 657. They were identified by Dong \& $\mathrm{Hu}$ (1991) with the IRAS sources $03549+5602$ and $22407+6008$, respectively, among many other sources displaying a very strong infrared excess, $V-[25] \geq$ 8 mag AS 78 is recognized as a photometric variable for the first time. A large near-IR excess radiation is detected in AS 78 and confirmed by new, higher-quality, data for MWC 657. Significant variations in the Balmer line profiles are detected for both objects. Modelling of the Balmer line profiles of AS 78 obtained in 1994 yields the following parameters of the star and its wind: $T_{\text {eff }} \sim$ $17000 \mathrm{~K}, \log L_{\mathrm{bol}} / L_{\odot} \sim 4.0, \dot{M} \sim 10^{-6} M_{\odot} \mathrm{yr}^{-1}$. The photometric and new spectroscopic data indicate that the star's $T_{\text {eff }}$ increases with time, while the stellar wind changes its structure. Distances towards both objects are estimated on the basis of their radial velocities and the galactic rotation curve. Consideration of different options about the nature and evolutionary state of both objects leads us to suggest that they are binary systems

Send offprint requests to: A.S. Miroshnichenko, e-mail: anatoly@physics.utoledo.edu

* Table 9 is only available in electronic form at the CDS via anonymous ftp (130.79.128.5) or via http://cdsweb.u-strasbg.fr/Abstract.html
\end{abstract}

containing a B-type intermediate-luminosity star and a gaseous disk around the less luminous component.

Key words: techniques: photometric - techniques: spectroscopic - stars: emission-line - stars: circumstellar matter — stars: individual (AS 78, MWC 657)

\section{Introduction}

Catalogues of emission-line stars which were compiled decades ago (e.g., MWC, Merrill \& Burwell 1933; AS, Merrill \& Burwell 1950; LS, Stock et al. 1960) contain a large number of unstudied objects. For most of these objects, we only know that they display the $\mathrm{H} \alpha$ line in emission. The attention of researchers is usually attracted to these objects only when new information becomes available, such as photometric variability, or the detection of excess radiation in some spectral region. This is the case for the two stars in the present paper.

All early-type emission-line stars are surrounded by a significant amount of circumstellar gas (for instance, due to a stellar wind or accretion) and, as a consequence, display IR excesses due to free-free and free-bound radiation. Some are also surrounded by dusty envelopes, which 
can be either remnants of the protostellar collapse at the pre-main-sequence stage of evolution or can be formed in the outer parts of the wind for more evolved stars. If dust is present in the circumstellar environment, the IR excesses are more prominent, especially in the mid- and far-IR region, and in some cases may have been detected by the IRAS satellite. To identify such objects, crossidentification of the sources from the catalogue of galactic early-type emission-line stars by Wackerling (1970) with those of the IRAS Point Source Catalogue (PSC) was carried out by Dong \& Hu (1991).

These authors selected only objects with a very strong IR excess, $V-[25] \geq 8 \mathrm{mag}$, and found 187 such objects. Only about $30 \%$ of this sample consists of objects with a known nature: Herbig Ae/Be stars, LBVs, WolfRayet stars, symbiotic and VV Cep binary systems, and $\mathrm{H}$ II regions. Most of the other objects have not been investigated yet. Studying the spatial distribution of these objects, Dong \& Hu found them to belong to the extreme Population I and concluded that they provided a rather complete sample for further study of the evolution of preand post-main-sequence stars of intermediate and high masses with these properties. The selection criterion used was rather crude, but it enabled the authors to find objects in which the circumstellar processes are highly active. Thus, new information about the poorly-studied objects from this list is important for our understanding of the evolution of stars and their envelopes.

In 1993 we began a study of a subsample of Dong \& Hu's list which contains most of their northern objects (north of $-15^{\circ}$ declination). These objects may display similar properties while having quite different natures and evolutionary states. Because of that, we have been grouping them on the basis of either similar observational characteristics or closeness in their physical parameters. Our first results were briefly presented at the Workshop on $\mathrm{B}[\mathrm{e}]$ stars (Kuratov et al. 1998; Sheikina \& Miroshnichenko 1998).

In the course of this study we obtained photometric and spectroscopic observations of two poorly-studied objects, AS 78 and MWC 657. Analysis showed that their properties are similar to those of $\mathrm{B}[\mathrm{e}]$ stars with hot circumstellar dust (Miroshnichenko et al. 1999). Our previous study of MWC 657 (Miroshnichenko et al. 1997) was limited to only photometric data and resulted in rough estimates of the star's fundamental parameters. Here we present the results of our spectroscopic and photometric analyses for both objects, including estimates of the main characteristics of the stars and their surrounding circumstellar matter. The observations are described in Sect. 2, results and discussion in Sect. 3, and our conclusions are given in Sect. 5 .
Table 1. Spectroscopic observations of AS 78 and MWC 657

\begin{tabular}{rccclr}
\hline Object & $\begin{array}{c}\text { JD } \\
\text { 2400000+ }\end{array}$ & Obs. & $\begin{array}{c}\text { Exp.t } \\
\text { min }\end{array}$ & $\begin{array}{l}\text { Range } \\
\AA\end{array}$ & $\begin{array}{r}\Delta \lambda \\
\AA\end{array}$ \\
\hline AS 78 & 49358.32 & SAO & 32 & $3530-5530$ & 4 \\
AS 78 & 49358.34 & SAO & 32 & $4930-6950$ & 4 \\
AS 78 & 49374.20 & SAO & 24 & $3895-4920$ & 2 \\
AS 78 & 49374.22 & SAO & 24 & $5705-6780$ & 2 \\
AS 78 & 51388.49 & SAO & 80 & $4540-7761$ & 0.6 \\
AS 78 & 51419.03 & Lick & a & $8000-13500$ & 14 \\
AS 78 & 51419.03 & Lick & a & $14000-24820$ & 36 \\
MWC 657 & 49592.58 & OHP & 60 & $3900-6815$ & 0.2 \\
MWC 657 & 49941.59 & OHP & 60 & $3900-6815$ & 0.2 \\
MWC 657 & 51066.72 & DSO & 48 & $3800-5600$ & 3.6 \\
MWC 657 & 51186.16 & SAO & 60 & $4735-6800$ & 0.6 \\
MWC 657 & 51542.50 & DSO & 60 & $3800-5600$ & 3.6 \\
\hline
\end{tabular}

The observatory name is listed in Col. 3, the spectral resolution in Col. 6.

a See text for the explanations.

\section{Observations}

The $\log$ of spectroscopic observations is presented in Table 1. The low-resolution observations of AS 78 were obtained on 1994 January 5 and 21 at the 6 -m telescope of the Special Astrophysical Observatory (SAO) of the Russian Academy of Sciences with a photoelectric TVscanner (Balega et al. 1979) mounted at the Nasmyth focus. The high-resolution spectrum of AS 78 was obtained on 1999 July 28 at the same telescope with the échelle-spectrometer PFES (Panchuk et al. 1998) and a $1140 \times 1170$ pixel CCD detector.

The infrared observations of AS 78 were acquired on 1999 August 28 with the $3-\mathrm{m}$ Shane reflector of Lick Observatory and the Aerospace near-infrared imaging spectrograph. The spectrograph, which is described in more detail by Rudy et al. (1999), uses two channels to provide wavelength coverage from 0.8 to $2.5 \mu \mathrm{m}$. The blue $(0.8-1.4 \mu \mathrm{m})$ and red $(1.4-2.5 \mu \mathrm{m})$ spectra, which are acquired simultaneously, are each combinations of 6 individual spectral segments taken at different wavelength positions. Of the 6 positions, there are 3 that are well separated in wavelength; for each of these, 2 distinct positions are used that are separated by 2.5 pixels. The latter provides both oversampling and protection from dropouts due to bad pixels. Each of the 6 individual spectral segments are in turn formed from 4 individual spectra, each of 5 seconds duration. These spectra are taken at two separate locations along the slit ("plus beam" and "minus beam") in the following sequence: plus, minus, minus, plus. The spacing along the slit was $20^{\prime \prime}$ and the slit was oriented North-South.

A $2^{\prime \prime}$ slit was employed for the observations of AS 78 and its calibrator star HR 1276, an F5 dwarf. The instrumental response and most of the effects of atmospheric absorption were removed by dividing the spectrum of AS 78 by that of the calibrator. To remove 

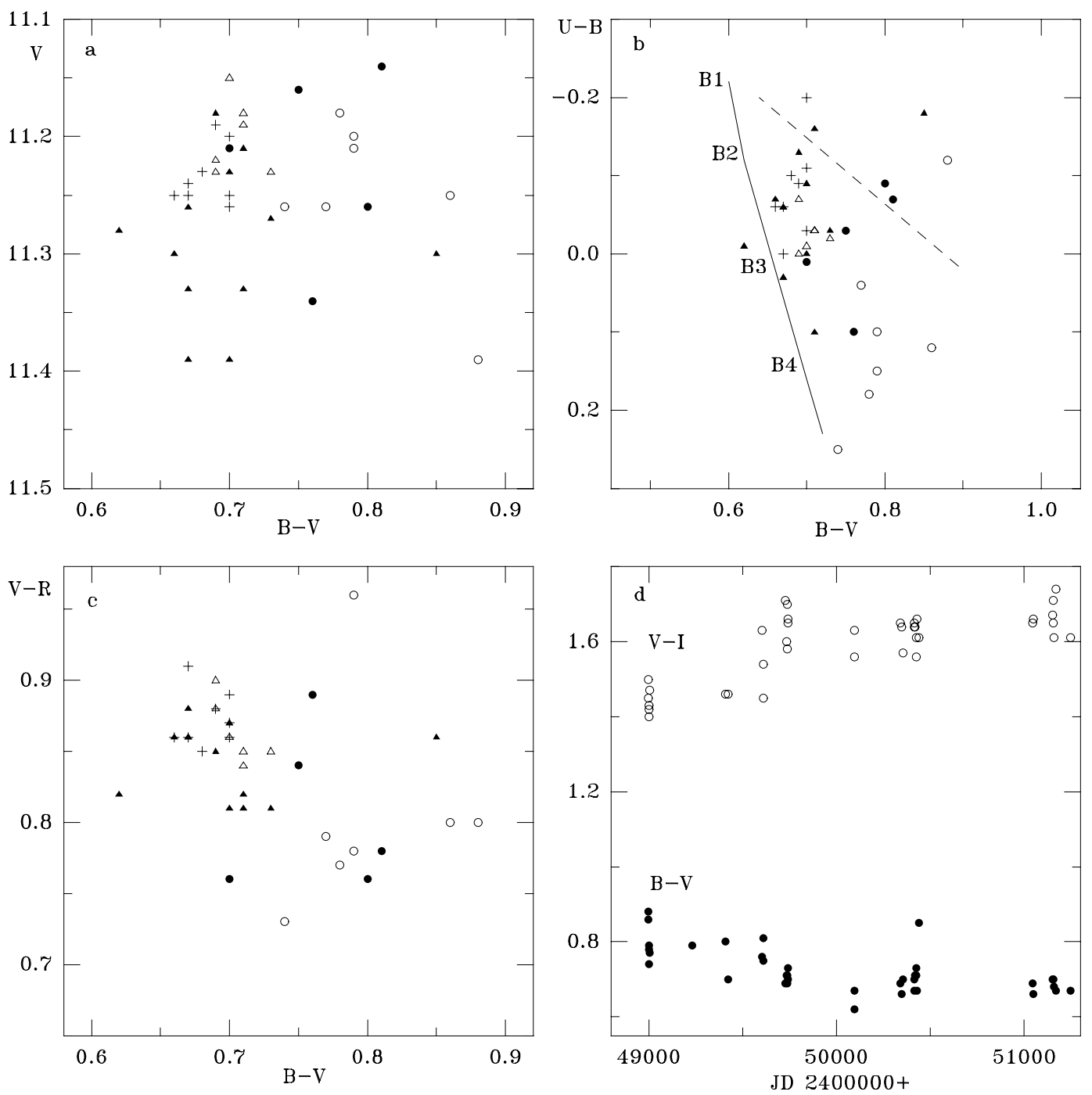

Fig. 1. Brightness and colour variations of AS 78. a) Colour-magnitude diagram; b) and c) colour-colour diagrams; d) temporal variations of $B-V$ (filled circles) and $V-I$ (open circles). Different symbols in panels a), b), and c) represent data obtained in different years: 1993 (open circles), 1994 (filled circles), 1995 (open traingles), 1996 (filled triangles), 1998/9 (pluses). The solid line in panel b) shows intrinsic colour-indices of B-type dwarfs from Strajzhys (1977) shifted along the interstellar reddening vector $E_{U-B} / E_{B-V}=0.84$ (dashed line) at $E_{B-V}=0.85$

the intrinsic spectrum of the calibrator from this ratio, a model from Kurucz (1994) appropriate to an F5 V star was used. The absolute flux level was set from the $K$-magnitude (5.17) of HR 1276. The $K$-magnitude was derived from the $V$-magnitude tabulated in the Bright Star Catalog (Hoffleit \& Jaschek 1982) and the nominal $V-K$ colour for an F5 dwarf given by Koornneef (1983). Using this absolute calibration, we estimated the star's brightness in the JHK bands. The results are listed in Table 2 and are in good agreement with the CST data for the $J$ and $H$ bands. The continuum flux at $0.9 \mu \mathrm{m}\left(1.110^{-16} \mathrm{~W} \mathrm{~cm}^{-1} \mu \mathrm{m}^{-1}\right)$ agrees well with the results of our $I$-band photometry $\left(\bar{I}=9.6 \mathrm{mag}\right.$ that corresponds to $1.210^{-16} \mathrm{~W} \mathrm{~cm}^{-1} \mu \mathrm{m}^{-1}$ according to Strajzhys 1977).
The low-resolution optical spectra of MWC 657 were obtained on 1998 September 10 and 1999 December 30 at the 0.8-m telescope of the Dark Sky Observatory (DSO) of Appalachian State University. We used the Gray/Miller spectrograph and a 600 lines $\mathrm{mm}^{-1}$ grating in the first order, with a Tektronics $1024 \times 1024$ thinned, back-illuminated CCD. These spectra have a spectral range of $3800-5600 \AA$, and a resolving power $R$ of about 1300 (3.6 $\AA / 2$ pixels). Two high-resolution spectra of MWC 657 were taken on 1994 August 28 and 1995 August 12 with a fiber-fed spectrograph ÉLODIE at the 1.93-m telescope of the Observatoire de Haute-Provence (south of France), in the framework of an extended search for spectroscopic binaries among young early-type emission-line stars (Corporon \& Lagrange 1999). Another 


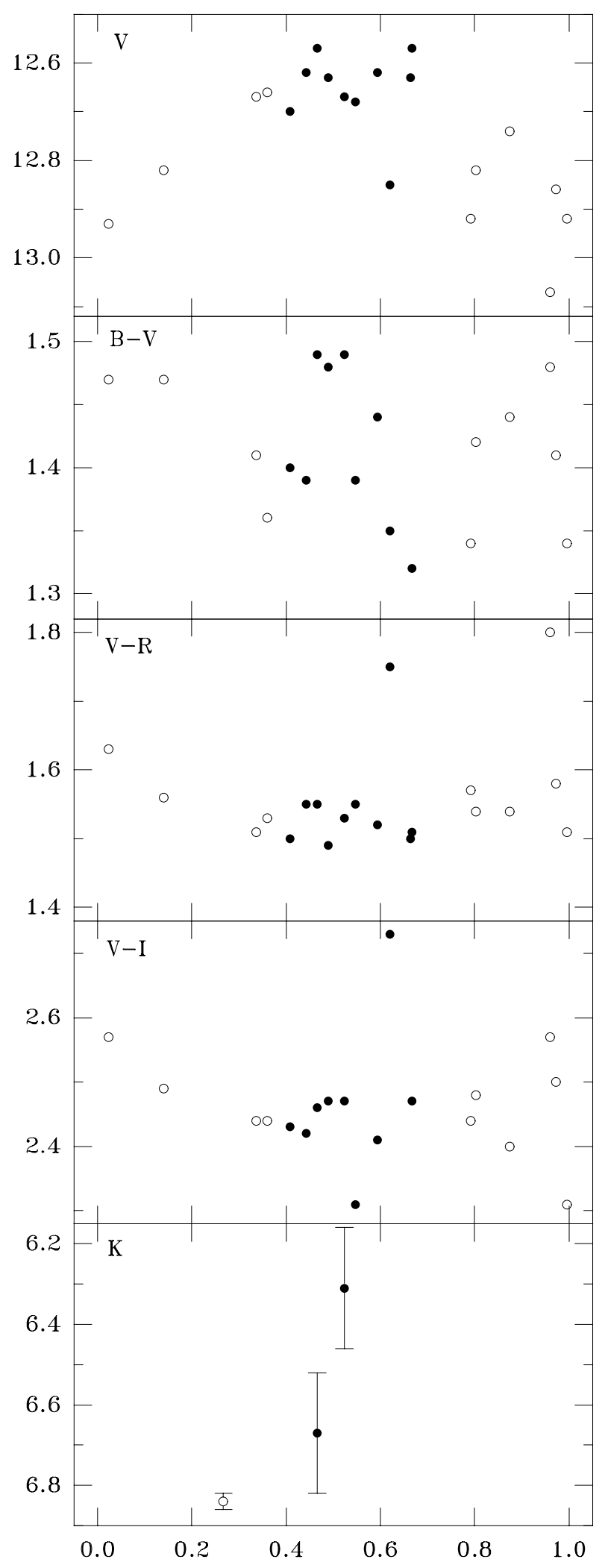

Fig. 2. Brightness and colour variations of MWC 657 folded onto the 86 day period. The 1996 data are marked by filled circles, while those of 1998 are open circles high-resolution optical spectrum of MWC 657 was obtained on 1999 January 7 at the SAO 6-m telescope with PFES.

The simultaneous $U B V R I H K$ observations in the Johnson photometric system were obtained between January 1993 and March 1999 with two 1-m telescopes at the Assy and Tien-Shan Observatories of the Fesenkov Astrophysical Institute of the National Academy of Sciences in Kazakhstan, equipped with a two-channel photometer-polarimeter (Bergner et al. 1988). The 26" diaphragm and a $85 \mathrm{~Hz}$ chopper throw of $84^{\prime \prime}$ in the north-south direction were used in all the runs. The results of the optical photometry are presented in Table 2 for AS 78 and in Table 6 for MWC 657. Typical errors of these observations do not exceed $0.03 \mathrm{mag}$ (0.05 mag in the $U$-band for MWC 657), and also include those of the transformation from the instrumental to the standard photometric system. The data with larger errors are marked with a colon. Only four $K$-band and one $H$-band observations were obtained for $\mathrm{AS} 78$, as its brightness turned out to be very close to the limiting magnitude of the equipment. These results are shown in Table 3 and discussed in Sect. 3.1.1. The results of our first $U B V R I K$ photometric observations of MWC 657 obtained in 1996 September-December were presented by Miroshnichenko et al. (1997).

On 1999 September 17 we obtained $J H K$ observations of both stars at the 1.55-m Carlos Sánchez Telescope (CST), operated by the Instituto de Astrofísica de Canarias at the Spanish Observatorio del Teide (Tenerife, Spain). We used a CVF infrared spectrophotometer equipped with an InSb photovoltaic detector, operating at the temperature of liquid nitrogen, with a photometric aperture of $15^{\prime \prime}$ and a chopper throw of $30^{\prime \prime}$ in the RA direction to subtract the contribution from the background sky. The Teide photometric system is described in Arribas and Martínez-Roger (1987), as well as its relations with other standard photometric systems. Since this system is very close to the Johnson system used at Tien-Shan, no additional corrections have been applied to the CST data. For the flux calibration we used several standard stars taken from the list given by Koornneef (1983), observed at different air masses to determine the atmospheric extinction in each filter during the night of the observation. Each star was observed 4 times in each band. The averaged data for AS 78 are included in Table 3, while those for MWC 657 are presented in Sect. 3.1.2.

On 2000 February 25 and 26, we obtained IR observations of AS 78 at the 3-m NASA IRTF, equipped with a single-element gallium-doped germanium bolometer, at Mauna Kea (Hawaii, U.S.A.). The photometric aperture of $10^{\prime \prime}$ and an $11 \mathrm{~Hz}$ chopper throw of $15^{\prime \prime}$ in the N-S direction was used. The measurements in the broad-band $K L M$ filters were taken on the first night, while both the broad-band $K L M N$ and narrow-band filters centered at 8.7, 9.8, 11.6, and $12.5 \mu \mathrm{m}$ were used on the second night. 
The accuracy of the measurements for AS 78 was close to 1 per cent in the broad-band filters, and about 4 per cent in the narrow-band ones. The magnitudes for HR 1457 and HR 1641 from the IRTF standard stars list were used to calibrate the $K L M$-data, while the results of Hammersley et al. (1998) obtained at IRTF for HR 2077 were used for the $N$-band and narrow-band data. The mean resulting accuracy of the object's magnitudes after calibration is 2 per cent for the broad-band and 5 per cent for the narrow-band data. Our IRTF data are listed in Table 4.

\section{Results}

\subsection{Photometric data}

\subsubsection{AS 78}

AS 78 (Merrill \& Burwell 1933) $=$ LS $\mathrm{I}+56^{\circ} 96$ is an 11-mag object in Camelopardalis which was identified by Miller \& Merrill (1951) as a Be star. Only one other $U B V$ observation is found in the literature (Haug 1970). Dong $\& \mathrm{Hu}$ (1991) identified AS 78 with the IRAS PSC source $03549+5602$, with fluxes at 12,25 , and $60 \mu \mathrm{m}$ of $3.75,3.13$, and $0.57 \mathrm{Jy}$ respectively. These fluxes indicate a strong IR excess with respect to the radiation of a hot star (see Fig. 11), that might be due the presence of circumstellar dust or a cool companion. The absence of spectral features of a late-type star in the spectrum of AS 78 and the large near-IR colour-indices (see Sect. 3.2.1) suggest that if there is a secondary component in the system, it is not responsible for the bulk of the visible and IR flux. Furthermore, the IRAS data are in agreement with the presence of circumstellar dust in the system (see Sect. 3.3).

Recently AS 78 was detected by the MSX satellite in the course of a mid-IR galactic plane survey (MSX5C-G146.9227+02.3139, Egan et al. 1999). Its fluxes were measured in three bands centered at 8.28 , 12.13 , and $14.65 \mu \mathrm{m}(3.69 \pm 0.18,3.06 \pm 0.23$, and $2.87 \pm 0.18 \mathrm{Jy}$ respectively). The $12-\mu \mathrm{m}$ IRAS, MSX, and IRTF fluxes agree with each other within a $3 \sigma$ range. The smooth flux decrease with wavelength in this region suggests that the $10 \mu \mathrm{m}$ silicate feature is probably weak or not present in the spectrum (see Fig. 11). Furthermore, a steep decrease in the IR flux toward longer wavelengths implies a large density gradient in the dust envelope and/or a small size. It might indicate that the dust formation process started recently and the dust is concentrated mainly close to the star.

No previous near-IR observations for this star have been reported in the literature. Our measurements (see Tables 3 and 4 ) show that a significant excess radiation has been reliably detected in this spectral range. Note that the CST $K$-magnitude is nearly 1 mag fainter than those of the other dates. This cannot be due to clouds, which were noticed nearly an hour after the observation of
AS 78, because the measurements in all three bands were repeated several times in different sequences, and the $J$ and $H$-band CST magnitudes are in good agreement with those obtained at Lick 3 weeks earlier. Nor do we believe that this discrepancy is due to the small diaphragm size used at CST, because the near-IR emission is due to hot dust which is located close to the star; $15^{\prime \prime}$ corresponds to $0.2 \mathrm{pc}$ at the distance of AS 78 (see Sect. 3.2.1) and certainly includes all the hot dust and even most of the cooler part of the dusty envelope. Our IRTF measurements through a $10^{\prime \prime}$ aperture confirm this suggestion. In any case, our near-IR data indicate that there is a noticeable variability at least in the $H$ - and $K$-bands.

In order to estimate the strength and shape of the nearIR excess we adopt $\overline{V-K}=4.8 \mathrm{mag}, \overline{J-H}=1.1 \mathrm{mag}$, and $\overline{H-K}=1.2 \mathrm{mag}$ (from Table 3 ), which correspond to the dereddened values of $2.4,0.8$, and $1.0 \mathrm{mag}$, respectively, using $E_{B-V}=0.9 \mathrm{mag}$ derived below. This is in agreement with the above suggestion about the presence of the circumstellar dust (see Fig. 11).

Our optical photometry (38 observations from more than 6 years) shows that the star's brightness varies with an amplitude of $0.25 \mathrm{mag}$ (see Table 2). The $U-B$ and $B-V$ colour-indices display a positive correlation with each other and a negative correlation with $V-R$ and $V-I$ (Fig. 1). The temporal trend direction in the $U-B$ vs. $B-V$ plane (see Fig. 1b) is closer to the line of the stellar intrinsic colour-indices (Strajzhys 1977) than to the interstellar reddening vector. This suggests that these colour variations are caused by changes in the star's parameters rather than by those of the circumstellar matter. The object's strong emission-line spectrum implies a certain contribution of the latter to the brightness and colour-indices. However, this contribution is probably small, since our results for the interstellar reddening derived from the photometry and spectroscopy (Sect. 3.2.1) are very close to each other.

The colour-indices $(U-B, B-V)$ change between $(-0.10,0.67)$ and $(0.15,0.77)$. These extreme values correspond to the spectral types B2 and B4 respectively (if we assume luminosity types $\mathrm{V}$ or III) with essentially the same reddening, $E_{B-V}=0.90 \pm 0.05$ mag. The spectral type interval would be B7 to B9.5, assuming that the star is a supergiant. These estimates were obtained using the ratio of total to selective extinction $R=3.1$, and $E_{U-B} /$ $E_{B-V}=0.84$, which was derived from the data listed in Table 5. A small ( $\sim 0.1 \mathrm{mag})$ excess radiation in the $R$ and $I$ bands is seen for all suggested luminosity types after dereddening. As the spectral type becomes earlier, $V-R$ and $V-I$ increase from 0.75 and 1.4 to 0.9 and $1.7 \mathrm{mag}$, respectively, yielding an increase in the red-excess radiation. Temporal evolution of the colour-indices shows that both $U-B$ and $B-V$ decrease with time, while both $V-R$ and $V-I$ increase (see Fig. 1d). This indicates that both the star's temperature and the red-excess radiation, which is most likely due to free-free radiation, increase 
Table 2. Optical photometry of AS 78 at the Tien-Shan Observatory

\begin{tabular}{crrrrrrrrrcc}
\hline JD 2400000+ & $V$ & $U-B$ & $B-V$ & $V-R$ & $R-I$ & JD $2400000+$ & $V$ & $U-B$ & $B-V$ & $V-R$ & $R-I$ \\
\hline a & 11.26 & -0.07 & 0.71 & - & - & 50097.19 & 11.26 & -0.06 & 0.67 & 0.86 & 0.77 \\
48997.15 & 11.25 & 0.12 & 0.86 & 0.80 & 0.70 & 50340.47 & 11.18 & -0.13 & 0.69 & 0.85 & 0.80 \\
48998.11 & 11.39 & -0.12 & 0.88 & 0.80 & 0.65 & 50348.48 & 11.30 & -0.07 & 0.66 & 0.86 & 0.78 \\
49000.10 & 11.21 & 0.15 & 0.79 & 0.78 & 0.65 & 50353.48 & 11.23 & -0.09 & 0.70 & 0.81 & 0.76 \\
49001.14 & 11.18 & 0.18 & 0.78 & 0.77 & 0.65 & 50415.27 & 11.39 & 0.00 & 0.70 & 0.87 & 0.77 \\
49002.12 & 11.26 & 0.25 & 0.74 & 0.73 & 0.67 & 50416.23 & 11.39 & 0.03 & 0.67 & 0.88 & 0.77 \\
49005.13 & 11.26 & 0.04 & 0.77 & 0.79 & 0.68 & 50417.23 & 11.33 & -0.16 & 0.71 & 0.82 & 0.82 \\
49232.47 & 11.20 & 0.10 & 0.79 & 0.96 & 0.95 & 50424.21 & 11.27 & -0.03 & 0.73 & 0.81 & 0.75 \\
49409.08 & 11.26 & -0.09 & 0.80 & 0.76 & 0.70 & 50426.27 & 11.21 & 0.10 & 0.71 & 0.81 & 0.80 \\
49423.11 & 11.21 & 0.01 & 0.70 & 0.76 & 0.70 & 50429.26 & 11.33 & -0.06 & 0.67 & 0.86 & 0.80 \\
49605.38 & 11.34 & 0.10 & 0.76 & 0.89 & 0.74 & 50439.21 & 11.30 & -0.18 & 0.85 & 0.86 & 0.75 \\
49610.39 & 11.16 & -0.03 & 0.75 & 0.84 & 0.70 & 51047.46 & 11.19 & -0.09 & 0.69 & 0.88 & 0.77 \\
49612.39 & 11.14 & -0.07 & 0.81 & 0.78 & 0.67 & 51051.44 & 11.25 & -0.06 & 0.66 & 0.86 & 0.80 \\
49729.10 & 11.23 & -0.07 & 0.69 & 0.88 & 0.83 & 51154.23 & 11.26 & -0.03 & 0.70 & 0.87 & 0.80 \\
49735.06 & 11.18 & -0.03 & 0.71 & 0.84 & 0.76 & 51156.25 & 11.20 & -0.20 & 0.70 & 0.89 & 0.82 \\
49738.11 & 11.19 & -0.03 & 0.71 & 0.85 & 0.73 & 51157.24 & 11.25 & -0.11 & 0.70 & 0.86 & 0.79 \\
49740.09 & 11.22 & 0.00 & 0.69 & 0.90 & 0.80 & 51159.24 & 11.23 & -0.10 & 0.68 & 0.85 & 0.76 \\
49741.09 & 11.23 & -0.02 & 0.73 & 0.85 & 0.80 & 51171.29 & 11.24 & -0.06 & 0.67 & 0.91 & 0.83 \\
49742.13 & 11.15 & -0.01 & 0.70 & 0.86 & 0.80 & 51247.15 & 11.25 & 0.00 & 0.67 & 0.86 & 0.75 \\
50096.15 & 11.28 & -0.01 & 0.62 & 0.82 & 0.74 & & & & & \\
\hline
\end{tabular}

a Data from Haug (1970).

with time. The latter fact might imply strengthening of the stellar wind.

A similar behaviour of $U-B$ and $B-V$ is a frequently observed phenomenon in classical Be stars (e.g., Harmanec 2000), which have significantly weaker emission-line strengths than those of AS 78, and very flattened circumstellar gaseous envelopes. A positive correlation at the same brightness level (as is seen in AS 78) is usually observed in the systems seen nearly edge- on and can be explained by a change of the Balmer discontinuity (Divan \& Zorec 1982). The latter is due to a change in the amount of circumstellar matter close to the star. Balmer line profiles of AS 78 suggest an insignificant flattening of its envelope (see Sect. 3.2.1) that may have the same impact on the color-indices. Therefore, while the stellar wind strengthening mentioned above may account for all the four color-index changes, since we do not have more detailed spectrophotometric information, we cannot rule out temperature changes as a possible contributor to the observed photometric behaviour.

Periodogram analysis of the optical brightness variations shows that the power spectrum contains a number of peaks of almost equal strength between 60 and 500 days. However, none of the possible periods displays a convincing phase curve.

To decide which luminosity type is more realistic for AS 78 we searched for photometric data of nearby stars in the sky. Several B-type supergiants found within an angular distance of $1^{\circ}$ (from the object) are listed in Table 5 . Despite the fact they have colour-indices close to those of
Table 3. Near-IR photometry of AS 78

\begin{tabular}{clllr}
\hline JD 2400000+ & $J$ & $H$ & $K$ & Obs. \\
\hline 50439.21 & & & $6.4 \pm 0.2$ & TS \\
51047.46 & & & $6.57 \pm 0.07$ & TS \\
51051.44 & & $7.1 \pm 0.1$ & $6.16 \pm 0.07$ & TS \\
51247.15 & & & $6.51 \pm 0.07$ & TS \\
51419.03 & $8.8 \pm 0.1$ & $7.7 \pm 0.1$ & $6.3 \pm 0.1$ & L \\
51439.12 & $8.76 \pm 0.03$ & $7.75 \pm 0.02$ & $7.45 \pm 0.02$ & T \\
\hline
\end{tabular}

The observatory name is listed in Col. 5 (TS - Tien-Shan, L - Lick, T - del Teide).

AS 78 and similar reddenings, they are $1-3$ mag brighter than AS 78. This fact might imply that our object is a low- or an intermediate-luminosity star rather than a high-luminosity supergiant. The photometric data from Table 5 indicate that the interstellar extinction $\left(A_{V}\right)$ varies between 2.5 and $3.0 \mathrm{mag}$ beyond $\sim 2.5 \mathrm{kpc}$ from the Sun in this direction. The extinction for AS 78 we derived from the averaged $B-V$ is $A_{V}=2.7 \pm 0.2 \mathrm{mag}$. The above data on the nearby stars and the derived $A_{V}$ imply that distance of AS 78 is at least $2.5 \mathrm{kpc}$.

\subsubsection{MWC 657}

The results of a photometric study of MWC 657 have recently been presented by Miroshnichenko et al. (1997), who concluded that it is an early B-type star which is located above the main sequence and is surrounded by 
Table 4. IRTF photometry of AS 78

\begin{tabular}{ccccccccc}
\hline JD 2400000+ & $K$ & $L$ & $M$ & $N$ & 8.7 & 9.8 & 11.6 & 12.5 \\
\hline 51599.77 & 6.57 & 5.14 & 4.35 & & & & & \\
51600.75 & 6.57 & 5.08 & 4.29 & 2.71 & 2.74 & 2.49 & 2.43 & 2.24 \\
\hline
\end{tabular}

The effective wavelengths of the broad-band IRTF filters are $2.2 \mu \mathrm{m}(K), 3.45 \mu \mathrm{m}(L), 4.8 \mu \mathrm{m}(M)$, and $10.4 \mu \mathrm{m}(N)$.

Table 5. Photometry of stars in the vicinity of AS 78

\begin{tabular}{lrrrllll}
\hline Name & $V$ & $U-B$ & $B-V$ & Sp.T. & $A_{V}$ & $\begin{array}{c}D \\
\mathrm{kpc}\end{array}$ & $\begin{array}{c}\text { Ang. } \\
\circ\end{array}$ \\
\hline $\mathrm{BD}+55^{\circ} 837$ & 9.58 & -0.26 & 0.70 & B2 Ib & 2.69 & 3.5 & 0.3 \\
$\mathrm{BD}+55^{\circ} 838$ & 9.29 & -0.10 & 0.82 & B3 Ib & 2.97 & 2.5 & 0.5 \\
$\mathrm{BD}+56^{\circ} 873$ & 8.98 & -0.51 & 0.49 & O9.5 Ib & 2.50 & 3.4 & 0.6 \\
$\mathrm{BD}+56^{\circ} 868$ & 9.14 & -0.57 & 0.31 & B0.5 V & 1.63 & 1.7 & 0.7 \\
$\mathrm{HDE} 237213$ & 8.72 & -0.06 & 0.77 & B3 Ia & 2.82 & 4.0 & 0.7 \\
LS I+56 92 & 10.26 & -0.09 & 0.69 & B6 I & 2.36 & 5.0 & 1.0 \\
\hline
\end{tabular}

$D$ is the distance from the Sun, which was calculated using a luminosity calibration by Strajzhys \& Kurilene (1981), Ang. is the angular distance from AS 78.

an optically thin dusty envelope. Ten $U B V R I$ observations obtained by these authors revealed a variability of about $0.3 \mathrm{mag}$ in all bands. Our new optical photometric data (see Table 6) show that these variations are even larger and reach $\sim 0.6 \mathrm{mag}$ for the whole data set $(20$ observations during 2.2 years). The near-IR excess in MWC 657 has been detected by Miroshnichenko et al. (1997) on the basis of $2 K$-band measurements obtained on 1996 December 4 and $10(6.67 \pm 0.15$ and $6.31 \pm 0.15$, respectively). Recently we obtained new measurements on 1999 September 17 (JD 2451439.043) with the following results: $J=9.14 \pm 0.04, H=8.05 \pm 0.02, K=6.84 \pm$ 0.02. The $1999 K$-band brightness is lower than that of 1996 which we explain below. Nevertheless, the new data confirm the existence of the near-IR excess in the object's SED. Furthermore, MWC 657 was detected by the MSX satellite (MSX5-G107.6722+01.4002) in four bands centered at $8.28,12.13,14.65$, and $21.34 \mu \mathrm{m}$ with the fluxes $5.64 \pm 0.28,5.33 \pm 0.22,4.43 \pm 0.21$, and $4.33 \pm 0.45 \mathrm{Jy}$ respectively. The $12-\mu \mathrm{m}$ MSX flux of MWC 657 is nearly 10 per cent smaller than that of IRAS, which is about a $2.5 \sigma$ difference. The MSX fluxes indicate that the presence of the $10-\mu \mathrm{m}$ silicate feature in the spectrum of MWC 657 is questionable, as in the case of AS 78.

Despite the small amount of photometric data obtained, we attempted the Lomb-Scargle periodogram analysis of the optical light curves (Scargle 1982). The strongest peak in the power spectrum corresponds to a period of 86.2 days. The phase curves folded onto this period are presented in Fig. 2. It turned out that the 1996 and 1998 optical photometry was obtained in non-overlapping phase ranges. However, since the same equipment was used in both data sets, this fact shows that the variations were stable during at least 10 cycles.
It is seen in Fig. 2 that the object generally becomes bluer when it brightens, although there is a short period of reddening around phase 0.5 which is best seen in the $B-V$ colour. The $K$-band brightness seems to increase along with the optical brightness. At the same time, we cannot rule out the possibility that the photometric variations detected reflect different activity states of the object and are not periodic.

A search for photographic plates containing images of MWC 657 in the Sternberg Astronomical Institute (Moscow, Russia) archive revealed 85 such plates. The object's brightness was estimated by N.E. Kurochkin (priv. commun.) by eye. He found that it varied between $m_{\mathrm{pg}} 13.5$ and 14.5 in 1901-1968. Unfortunately, it turned out to be close to the plates' threshold, and the estimates have large errors. As a result, they do not show any variations with a period close to that of the photoelectric data. Nevertheless, the photographic data show no dramatic variations in the brightness of MWC 657.

Table 6. Optical photometry of MWC 657

\begin{tabular}{crrrrc}
\hline JD 2400000+ & $V$ & $U-B$ & $B-V$ & $V-R$ & $R-I$ \\
\hline 51053.39 & 12.92 & -0.14 & 1.34 & 1.57 & 0.87 \\
51054.26 & 12.82 & & 1.42 & 1.54 & 0.94 \\
51060.45 & 12.74 & & 1.44 & 1.54 & 0.86 \\
51073.27 & 12.93 & $-0.3:$ & 1.47 & 1.63 & 0.94 \\
51083.31 & 12.82 & -0.09 & 1.47 & 1.56 & 0.93 \\
51100.27 & 12.67 & 0.02 & 1.41 & 1.51 & 0.93 \\
51102.30 & 12.66 & $-0.1:$ & 1.36 & 1.53 & 0.91 \\
51154.11 & 13.07 & & 1.48 & 1.80 & 0.77 \\
51155.06 & 12.86 & 0.08 & 1.41 & 1.58 & 0.92 \\
51157.06 & 12.92 & $0.0:$ & 1.34 & 1.51 & 0.80 \\
\hline
\end{tabular}




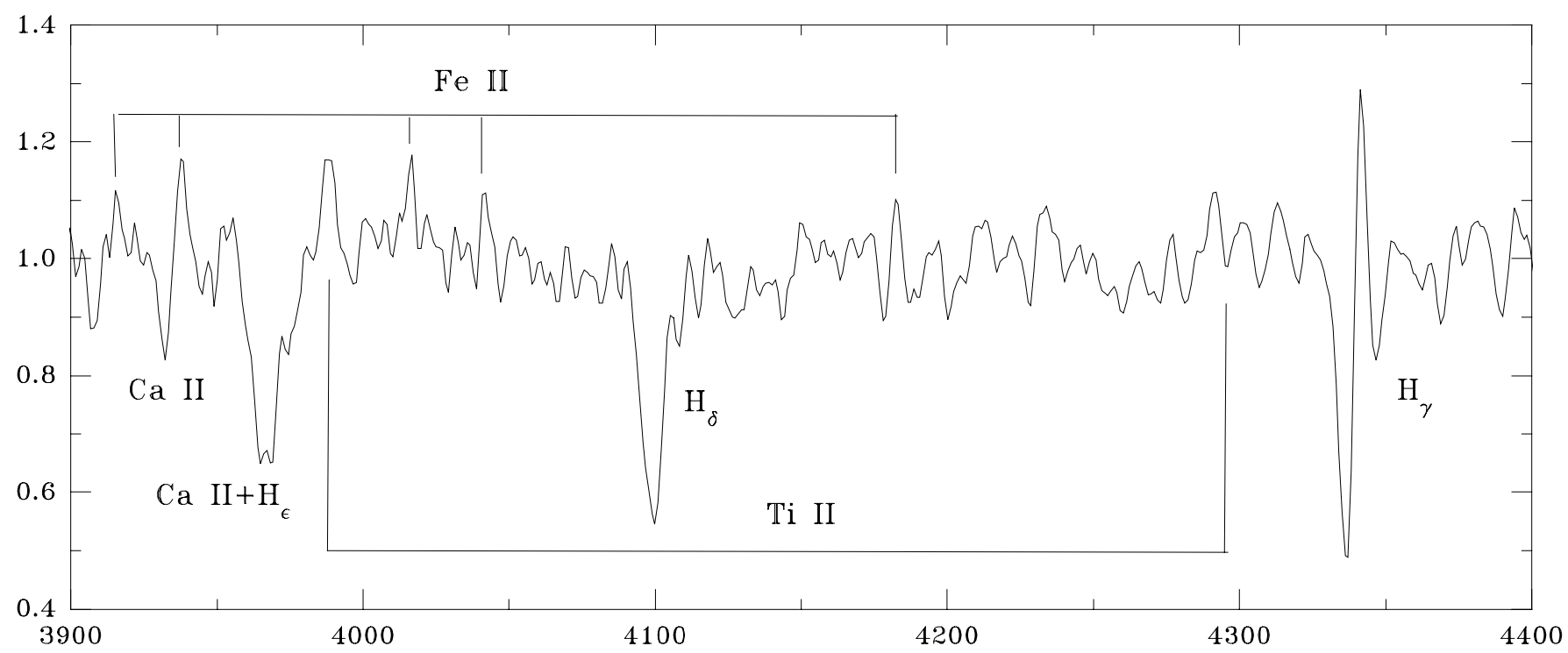

Fig. 3. The blue region of the low-resolution spectrum of AS 78. Wavelengths are given in $\AA$ while the intensities are normalized to the continuum

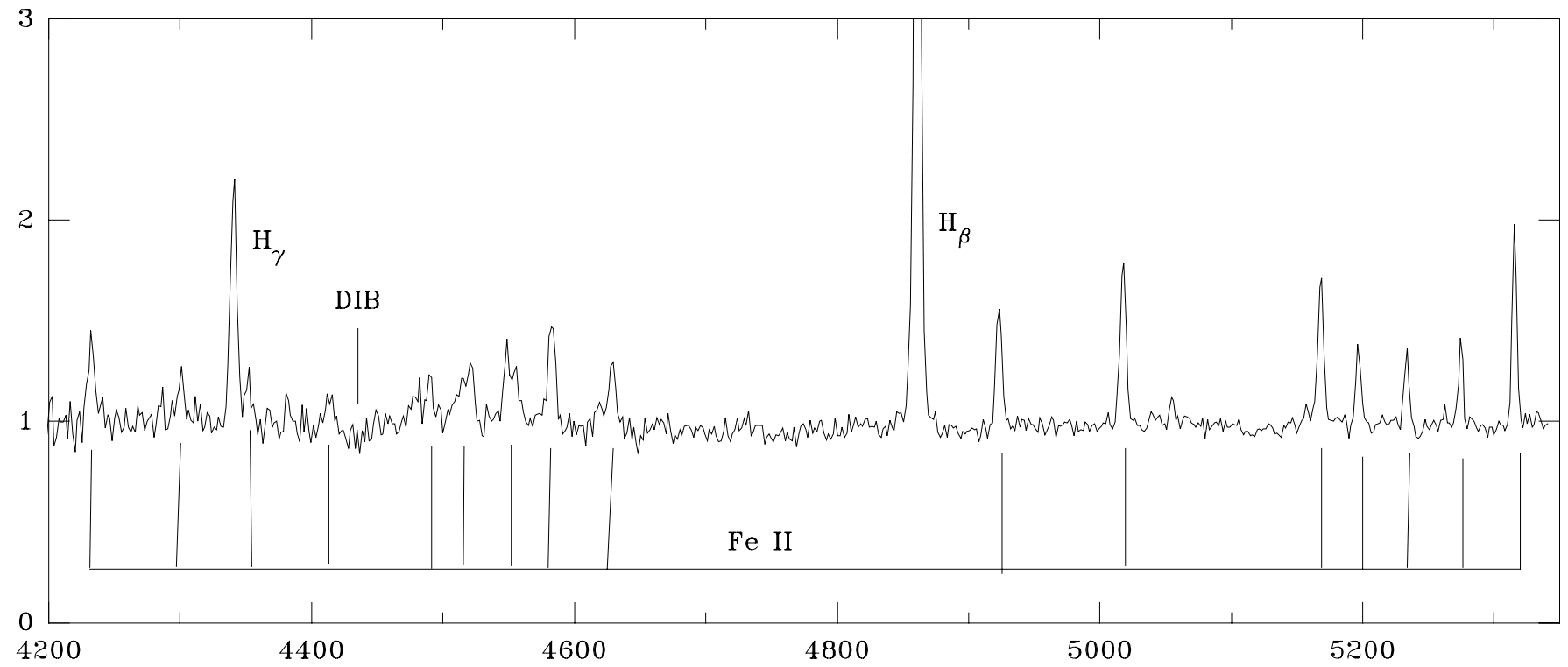

Fig. 4. A part of the low-resolution spectrum of MWC 657. Units of the wavelengths and intensities are the same as in Fig. 3

\subsection{Spectroscopic data}

The optical spectra of AS 78 and MWC 657 look very similar. However, the emission lines are stronger in MWC 657. Both objects show Balmer emission lines with very steep decrements, and a large number of $\mathrm{Fe}$ II lines in emission. The strongest Fe II lines (4923, 5018, and $5169 \AA$ ) have P Cyg-type profiles in AS 78 and in the SAO spectrum of MWC 657. Forbidden emission lines of $\mathrm{O}$ I are found in the spectra of both objects, while no [Fe II] lines are detected. The absorption features are mainly diffuse interstellar bands (hereafter DIBs). The only features which can be assigned to the photosphere are He I lines (and the Ne I $6402 \AA$ line in the spectrum of AS 78). However, in the OHP 1995 and SAO spectra of MWC 657 these are seen partly in emission. A catalog by Coluzzi (1993) was used to identify the spectral lines. Characteristics of the identified lines in the spectra of both objects are listed in Table 7 (Balmer lines only), Table 8 (lines in the near-IR spectrum of AS 78), and Table 9 (other lines in the optical region). For MWC 657 the information from the OHP 1994 and SAO spectra is given in Table 9, because the OHP 1995 spectrum was obtained with a lower signal-to-noise ratio. The most informative parts of the low-resolution spectra of both objects are shown in Fig. 3 

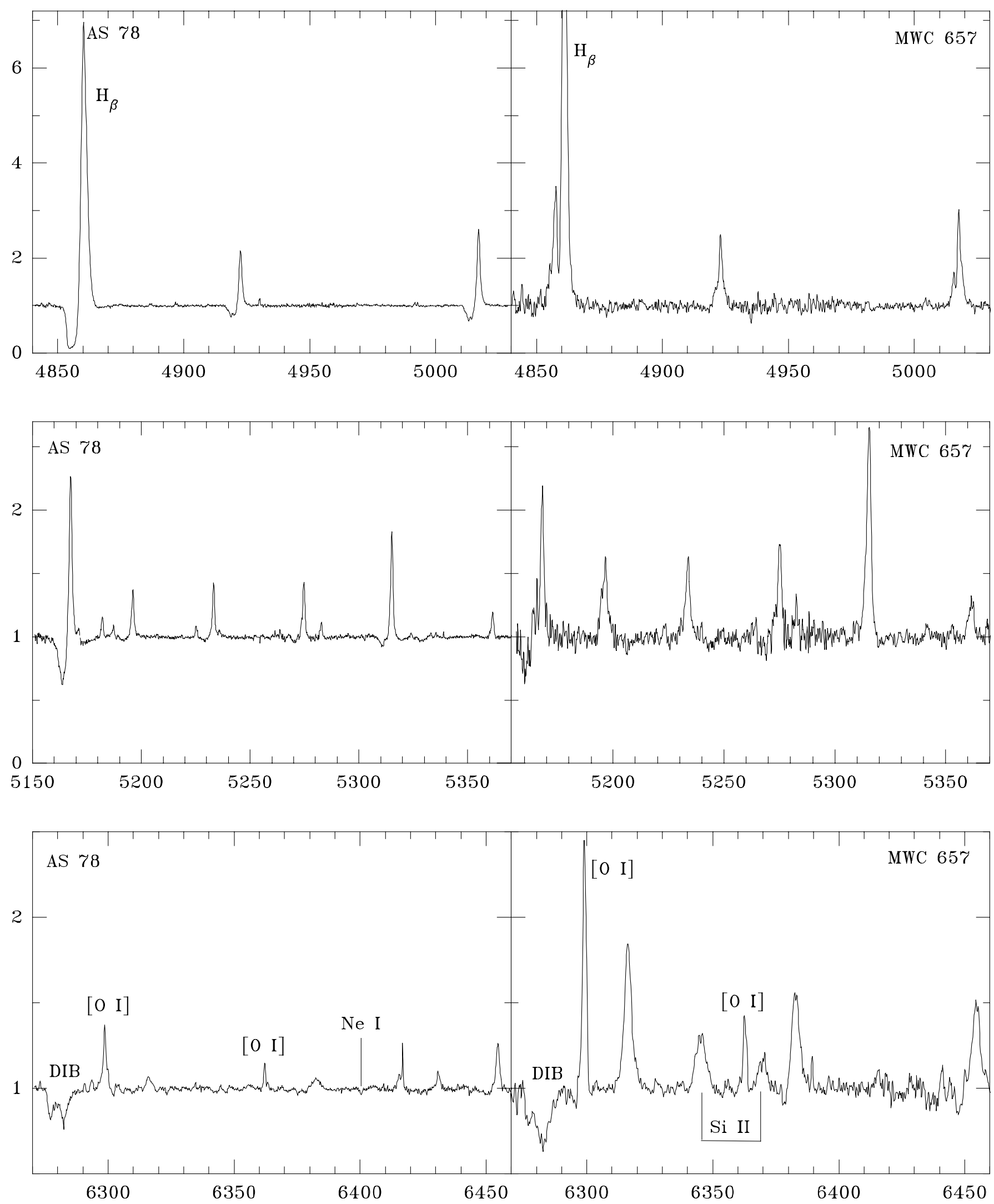

Fig. 5. Parts of the high-resolution SAO spectra of AS 78 and MWC 657. Unmarked lines are those of Fe II. Units of the wavelengths and intensities are the same as in Fig. 3 


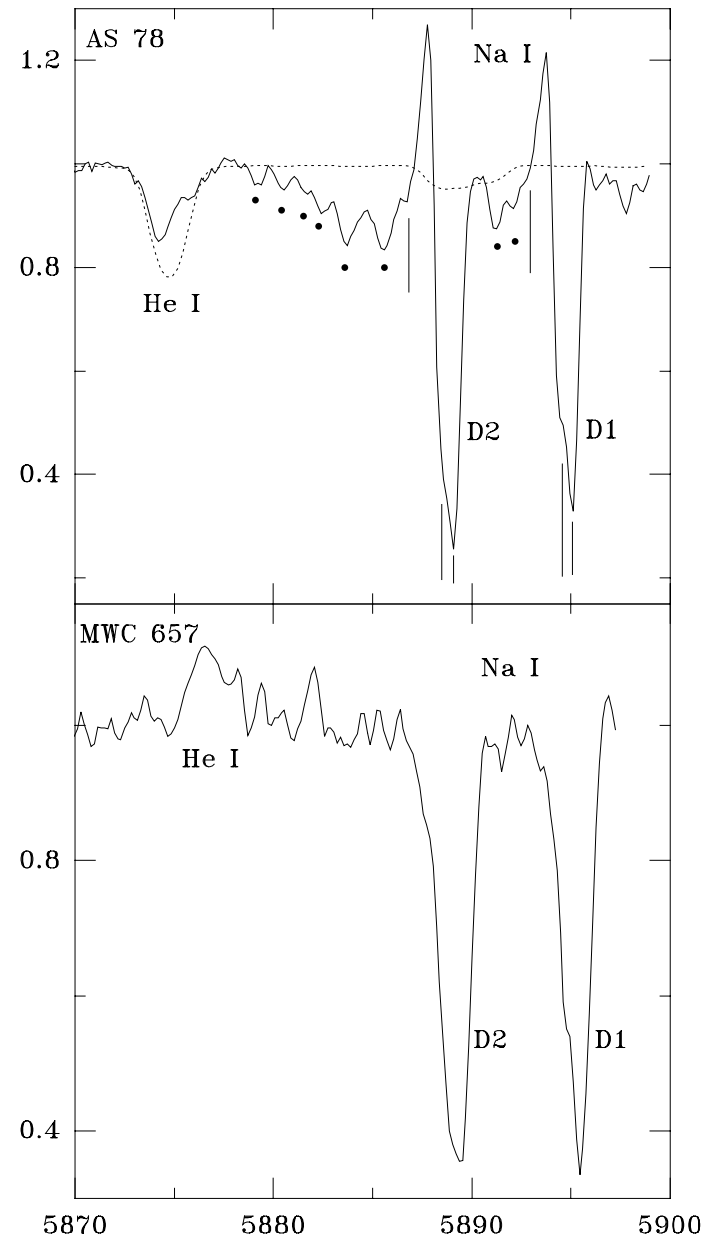

Fig. 6. The He I $5876 \AA$ and $\mathrm{Na}$ I $\mathrm{D}_{1,2}$ lines in the highresolution SAO spectra of AS 78 and MWC 657. The theoretical profile for $T_{\text {eff }}=17000 \mathrm{~K}, \log g=2.5$, and $v \sin i=$ $70 \mathrm{~km} \mathrm{~s}^{-1}$ is shown by a dotted line for AS 78. The absorption components of the $\mathrm{Na}$ I lines in the spectrum of AS 78 are marked by short solid lines, while the telluric water vapour absorption lines are marked by dots. Units of the wavelengths and intensities are the same as in Fig. 3

(AS 78) and 4 (MWC 657), while those of the high-resolution spectra are shown in Fig. 5 (both objects). An important spectral region containing the He I $5876 \AA$ line and the $\mathrm{Na} \mathrm{I} \mathrm{D}_{1,2}$ lines is presented in Fig. 6. The variations of the He I $5876 \AA$ line in the spectrum of MWC 657 are shown in Fig. 7. The theoretical He I line $5876 \AA$ profiles presented in Figs. 6 and 7 were calculated using the code Synspec (Hubeny et al. 1995) on the basis of the Kurucz (1994) model atmospheres and solar abundances.

Despite the above-mentioned similarities, the objects' spectra are different in details. Thus we will discuss the spectral features of these objects separately.

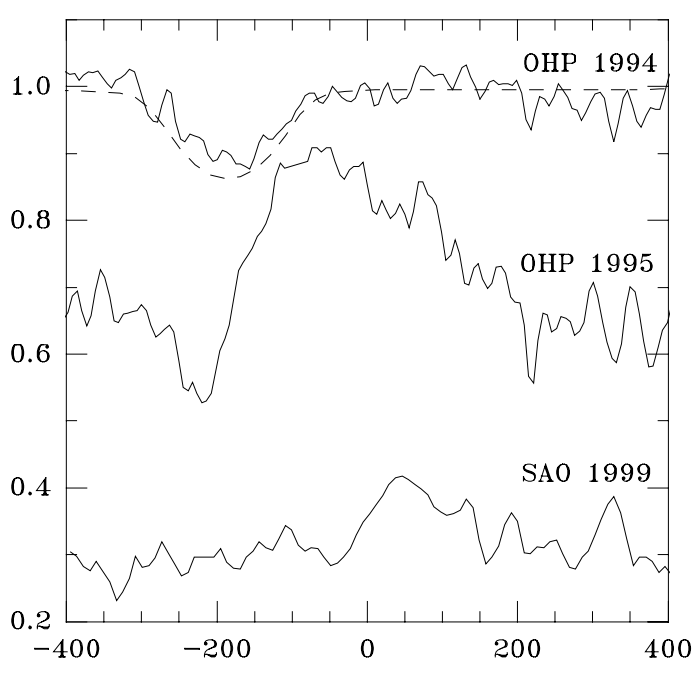

Fig. 7. The He I $5876 \AA$ line profile in the high-resolution spectra of MWC 657. The theoretical profile for $T_{\text {eff }}=15000 \mathrm{~K}$, $\log g=3.0$, and $v \sin i=100 \mathrm{~km} \mathrm{~s}^{-1}$ is shown by a dashed line. The velocities are given in $\mathrm{km} \mathrm{s}^{-1}$. The intensities are normalized to the continuum. The spectra are shifted with respect to each other by $0.35 I_{\text {cont }}$

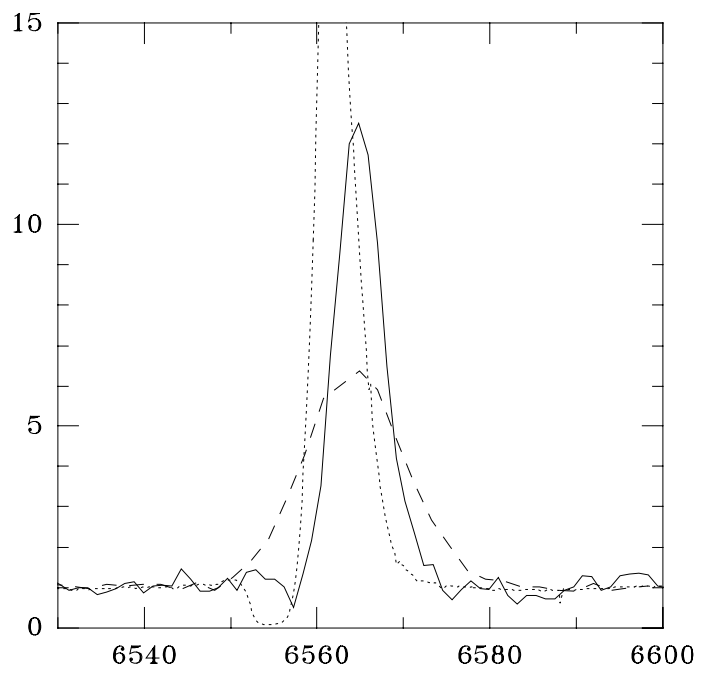

Fig. 8. The $\mathrm{H} \alpha$ line profiles in the spectrum of AS 78 obtained with different resolutions. The spectrum of 1994 January 5 is shown by a dashed line, that of 1994 January 21 by a solid line, while that of 1999 July 28 by a dotted line. Units of the wavelengths and intensities are the same as in Fig. 3

\subsubsection{AS 78}

The Balmer lines $(\mathrm{H} \alpha-\mathrm{H} \delta)$ have $\mathrm{P}$ Cyg-type profiles in both high- and low-resolution spectra of AS 78, indicating the presence of a strong stellar wind. The weak blueshifted peak and broad wings seen in the high-resolution $\mathrm{H} \alpha$ profile suggest a significant amount of electron scattering. The emission components of the higher members of the series are seen together with the broad photospheric lines (see Fig. 3). The equivalent width of the $\mathrm{H} \alpha$ line was nearly 
Table 7. Balmer lines in the spectra of AS 78 and MWC 657

\begin{tabular}{|c|c|c|c|c|c|c|c|c|c|}
\hline Name & Line & $V_{\text {blue }}$ & $I_{\text {blue }}$ & $V_{\text {abs }}$ & $I_{\mathrm{abs}}$ & $V_{\text {red }}$ & $I_{\text {red }}$ & $E W$ & Rem. \\
\hline \multirow[t]{7}{*}{ MWC 657} & $\mathrm{H} \alpha$ & -203 & 6.48 & -115 & 1.83 & -35 & 37.28 & 172.7 & OHP 1994 \\
\hline & & -249 & 8.22 & -118 & 1.46 & -36 & 46.68 & 198.0 & OHP 1995 \\
\hline & & -208 & 22.00 & -130 & 8.30 & $-30:$ & $\sim 80$ & & SAO $1999^{\mathrm{a}}$ \\
\hline & $\mathrm{H} \beta$ & -283 & 1.90 & -135 & 0.42 & +6 & 9.83 & 38.5 & OHP 1994 \\
\hline & & -238 & 1.46 & -125 & 0.46 & -21 & 8.96 & 33.3 & OHP 1995 \\
\hline & & -220 & 3.45 & $-150:$ & 1.30: & $-25:$ & 12.00: & 48.6 & SAO 1999 \\
\hline & $\mathrm{H} \gamma$ & -315 & 1.38 & -141 & 0.13 & +16 & 4.26 & 16.9 & OHP 1994 \\
\hline \multirow[t]{6}{*}{ AS 78} & $\mathrm{H} \alpha$ & & & & & & 6.4 & 77 & 1994 Jan. 5 \\
\hline & $\mathrm{H} \alpha$ & & & & & & 11.9 & 74 & 1994 Jan. 21 \\
\hline & $\mathrm{H} \beta$ & & & & & & 2.8 & 10 & 1994 Jan. 21 \\
\hline & $\mathrm{H} \gamma$ & & & & & & 1.4 & 5 & 1994 Jan. 21 \\
\hline & $\mathrm{H} \alpha$ & -570 & 1.18 & -350 & 0.06 & -23 & 29.00 & 117.5 & 1999 Jul. 28 \\
\hline & $\mathrm{H} \beta$ & & & -330 & 0.05 & -12 & 7.10 & 28.8 & 1999 Jul. 28 \\
\hline
\end{tabular}

The heliocentric radial velocities of the blue emission peak, central absorption, and red emission peak are listed in Cols. 3, 5, and 7 respectively; their intensities in continuum units in Cols. 4,6 and 8; the equivalent widths $(E W)$ are given in Col. 9 in $\AA$. The source of the data is listed in Col. 10. No velocity information is given for the low-resolution data.

a The red peak of the $\mathrm{H} \alpha$ line is saturated.

$75 \AA$ in 1994 and $117.5 \AA$ in 1999 . The central intensity of the Balmer lines strongly depends on the resolution (see Table 7 and Fig. 8). This is partly due to the narrow emission component of the line, but so remarkable a change in the line strength between 1994 and 1999 can also be explained by variations of the stellar wind. The change in the Balmer decrement $\left(\log I_{\mathrm{H} \alpha} / I_{\mathrm{H} \beta}=0.95\right.$ in 1994 and 0.69 in 1999) confirms the latter suggestion.

The sodium $\mathrm{D}_{1,2}$ lines consist of one emission and three absorption components (see Fig. 6). The strengths of the two deepest components, which are barely resolved at our resolution and certainly have an interstellar origin, are consistent with the high reddening evident from the photometry. Their radial velocities (hereafter $R V$ ), -4 and $-40: \mathrm{km} \mathrm{s}^{-1}$, suggest that the star belongs to the Perseus arm. Munch (1957) has shown that in this direction of the galactic plane such components are seen in the spectra of stars located farther than $2 \mathrm{kpc}$ from the Sun. The emission components of the D-lines seem to have P Cyg-type profiles, which are very similar to those of the strongest Fe II lines.

Nearly 10 DIBs are found in the spectrum of AS 78 . Since their fine structure is not resolved in our spectrum, we are only able to roughly estimate where they are formed. The mean DIBs $R V$ of about $-12 \mathrm{~km} \mathrm{~s}^{-1}$ is consistent with their formation between the Sun and Perseus arm. The strength of some DIBs in stellar spectra display a good correlation with the star's reddening (e.g. Herbig 1993). We use this property to obtain an independent estimate of $E_{B-V}$. The equivalent widths of the DIBs at 5780 and $5797 \AA, 0.43$ and $0.11 \AA$, respectively, correspond to $E_{B-V}=0.9$ (Herbig 1993), which is in good agreement with the dereddened optical colour-indices.

Only a few absorption lines of non-interstellar origin are seen in the spectrum of AS 78. The He I lines (4713, $5876,6678,7065$, and $10830 \AA$ ) are rather weak, while all except for the IR line, which is not resolved, show emission components superimposed on the underlying absorption line (see Fig. 6). Thus these lines are most likely affected by the envelope emission and cannot be used to derive the star's spectral type. At the same time, the line wings suggest a rotational velocity of nearly $70 \mathrm{~km} \mathrm{~s}^{-1}$. Another photospheric line, Ne I $6402 \AA$, is also very weak $(E W=0.03 \AA$, see Fig. 5). It is much weaker than those of B-type supergiants, reviewed in Miroshnichenko et al. (1998), irrespective of their sub-type and the presence of emission lines. This fact may indicate that the luminosity of AS 78 is not very high; however, this line may also be contaminated with the envelope emission.

The systemic velocity may be estimated by measuring the $R V$ of the Fe II lines (e.g. Humphreys et al. 1989). The mean $R V$, determined by using $21 \mathrm{Fe}$ II lines, is $-41 \pm 1 \mathrm{~km} \mathrm{~s}^{-1}$ and corresponds to $D=2.9 \pm 0.1 \mathrm{kpc}$ from the Sun, which is based on the galactic rotation curve (Dubath et al. 1988). The mean $R V$ of the He I lines, $-35 \pm 2 \mathrm{~km} \mathrm{~s}^{-1}$, which may be less affected by the envelope velocity field, is close to the result obtained for the Fe II lines. However, since $R V$ were measured for only 
three He I lines (see Table 9), the distance estimate based on them may introduce a larger uncertainty. In any case, the spectroscopic result does not contradict the photometric distance estimate (see Sect. 3.1.1). Note that the distance $(2.9 \mathrm{kpc})$ towards $\mathrm{BD}+55^{\circ} 837$, the closest star to AS 78 in Table 5 (and which also has $R V=-40 \mathrm{~km} \mathrm{~s}^{-1}$, Rubin 1965), determined from the galactic rotation, is in good agreement with the spectroscopic estimate $(3.5 \mathrm{kpc})$. Assuming $D=2.9 \mathrm{kpc}$ for AS 78, $V=11.2 \mathrm{mag}, A_{V}=$ 2.6 mag and spectral type B3 (see Sect. 3.1.1), one can derive $M_{V}=-3.7 \mathrm{mag}$ and $\log L_{\mathrm{bol}} / L_{\odot}=4.0$. Thus, the luminosity of AS 78 turns out to be intermediate between those of luminosity classes II and III (Strajzhys \& Kurilene 1981), unless we significantly underestimated the distance towards it.

The low-resolution near-IR spectrum of AS 78 contains emission lines of neutral hydrogen, singly ionized nitrogen, calcium, oxygen, and iron (see Table 8 and Fig. 9). The only line with a strong absorption component identified in this spectrum is that of He I $1.083 \mu \mathrm{m}$, which also has a weak, but noticeable, emission component, fairly unusual at this resolution. The IR triplet of Ca II has an averaged intensity of 1.25 of the continuum flux. A weak $\operatorname{Br} \gamma$ and a few higher members of this series are detected in emission. The flux ratio of the $\mathrm{O}$ I lines at 8446 , 11287 , and $13164 \AA$ can be used for an independent interstellar reddening estimate by means of the method suggested by Rudy et al. (1991). This method assumes that an equal number of photons are generated in the 8446 and $11287 \AA$ lines by the $\mathrm{Ly}_{\beta}$ fluorescence mechanism, but corrects this ratio, by using the $13164 \AA$ line, for the additional photons generated in the $8446 \AA$ line by continuum fluorescence. The flux ratio observed in the spectrum of $\operatorname{AS} 78(0.344 / 0.322 / 0.039)$ gives $E_{B-V}=0.70 \pm 0.15$, which agrees with the above photometric estimate.

The continuum level in the blue part of the near-IR spectrum decreases with wavelength, while it increases in the red part. This fact implies a change in the continuum formation mechanism. Shortward of $1.2 \mu \mathrm{m}$ it is due to the radiation of the star and the stellar wind. Extrapolation of the blue continuum slope to the red part of the spectrum shows that the observed continuum is 1.7 times stronger in the $H$-band and 5 times stronger in the $K$-band. Such a sharp rise cannot be explained by the presence of a cool companion, but rather by radiation of the circumstellar dust.

\subsubsection{MWC 657}

In contrast with those of $\mathrm{AS} 78$, the Balmer lines of MWC 657 show a double-peaked structure with a deep central depression (see Fig. 10). Such behaviour suggests that the lines are formed in a dense circumstellar disk, which is viewed close to edge-on. The intensity ratio of the blue and red peaks $(V / R)$ in the $\mathrm{H} \beta$ line profile is nearly 1.5 times larger in the 1999 spectrum compared to those in $1994 / 5$. The same effect is probably observed in the $\mathrm{H} \alpha$ line as the blue peak strength in the 1999 spectrum is at least 3 times larger than that in $1994 / 5$, while this number should not be larger than 1.5 for the red peak (a rough estimate from the unsaturated part of the profile). The variations of the $\mathrm{H} \alpha$ and $\mathrm{H} \beta$ profiles in the spectrum of MWC 657 are shown in Fig. 10. The low-resolution DSO spectra reveal the presence of a DIB at $4430 \AA$ and a number of Fe II lines in the region blueward of $4735 \AA$, the blue edge of the high-resolution SAO spectrum (see Fig. 4). The Balmer lines up to $\mathrm{H} \epsilon$ are seen in pure emission; while the shortest wavelength Balmer lines observed, $\mathrm{H} 8$ and H9, partially fill-in their absorption cores.

Since the emission-line spectrum of MWC 657 is much stronger than that of AS 78, no reliable information about the star's parameters can be derived from the few He I lines detected in absorption. Moreover, in the 1995 spectrum a part of the He I $5876 \AA$ line is seen in emission, as well as both He I 5876 and $6678 \AA$ in the 1999 spectrum (see Fig. 7). The fact that no He II $4686 \AA$ line is present puts an upper limit of $26-2710^{3} \mathrm{~K}$ on the disk's excitation source temperature. On the other hand, the occasional emission in the He I lines implies that it is $\geq 15000 \mathrm{~K}$.

The presence of the strong DIBs and deep interstellar components of the $\mathrm{Na}$ I $\mathrm{D}_{1,2}$ lines is consistent with the high reddening estimated by Miroshnichenko et al. (1997). The equivalent widths of the DIBs at 5780 and $5797 \AA$, 0.51 and $0.15 \AA$ respectively, lead to an estimate of the interstellar reddening of $E_{B-V} \sim 1.1 \mathrm{mag}$, which is noticeably smaller than the $1.6 \mathrm{mag}$ derived from the observed and intrinsic (for an early B-type star) colour-indices. This discrepancy indicates that part of the reddening is circumstellar. Such circumstellar reddening is usually observed in classical Be stars (e.g. Waters et al. 1987). In the case of MWC 657, this points out that the circumstellar gas density distribution decreases more slowly with distance from the star than that of AS 78. The emission peak separation $\left(\sim 200 \mathrm{~km} \mathrm{~s}^{-1}\right)$ is typical for classical Be stars, which may have Keplerian disks (e.g. Hummel 2000). On the other hand, the latter display significantly weaker emission-line spectra, indicating that MWC 657 has a higher average disk density.

The mean RV determined from the Fe II line measurements is $-55 \pm 5 \mathrm{~km} \mathrm{~s}^{-1}$ in the OHP and SAO spectra. According to Dubath et al. (1988) this corresponds to a distance of $4.5 \pm 0.5 \mathrm{kpc}$, while the distance to the neighboring OB-association Cep OB1, which has an averaged RV of $-60 \mathrm{~km} \mathrm{~s}^{-1}$, is $3.5 \mathrm{kpc}$ (Humphreys 1978). The interstellar extinction law in the direction of MWC 657 suggests $D \sim 2-3 \mathrm{kpc}$ for the interstellar part of the object's reddening (Miroshnichenko et al. 1997). Thus, $D=3.5 \mathrm{kpc}$ seems to be a reasonable estimate for the distance towards MWC 657. The averaged optical brightness ( $V=12.8 \mathrm{mag})$, the minimum star's 
Table 8. Lines in the near-IR spectrum of AS 78

\begin{tabular}{|c|c|c|c|c|c|}
\hline$\lambda_{\text {lab }}$ & ID & $I / I_{\mathrm{c}}$ & $\lambda_{\text {lab }}$ & ID & $I / I_{\mathrm{c}}$ \\
\hline 8138.59 & [Ti II] (7F) & 1.07 & 10049.38 & $\mathrm{P} 7$ & 1.45 \\
\hline 8203.572 & $\mathrm{P}^{\mathrm{a}}$ & 1.10 & 10459.79 & N II (11) & 1.09 \\
\hline 8446.35 & O I (8) & 1.27 & 10494.00 & {$\left[\begin{array}{ll}\mathrm{Cr} & \mathrm{II}\end{array}\right](28 \mathrm{~F}) ?$} & 1.07 \\
\hline 8498.018 & Ca II (2) & 1.17 & 10501.50 & Fe II & 1.05 \\
\hline 8542.089 & Ca II (2) & 1.30 & 10683.08 & $\mathrm{C}_{\mathrm{I}}$ & 1.20 \\
\hline 8598.394 & $\mathrm{P} 14$ & 1.10 & 10830.34 & He I $(1)^{\mathrm{b}}$ & 0.72 \\
\hline 8662.140 & Ca II $(2)+\mathrm{P} 13$ & 1.25 & 10862.64 & Fe II & 1.08 \\
\hline 8750.00 & $\mathrm{P} 12$ & 1.10 & 10938.09 & P6 & 1.65 \\
\hline 8862.787 & P11 & 1.13 & 11125.44 & Fe II & 1.06 \\
\hline 9017.911 & P10 & 1.20 & 11287.0 & $\mathrm{O}_{\mathrm{I}}$ & 1.27 \\
\hline 9061.33 & Fe II $(71)$ & 1.10 & 11750.0 & $\mathrm{C}_{\mathrm{I}}$ & 1.12 \\
\hline 9229.017 & P9 & 1.25 & 12818.05 & $\mathrm{P} 5$ & 1.95 \\
\hline 9381.78 & [Cr II $](23 \mathrm{~F})$ & 1.17 & 13164.8 & $\mathrm{O}$ I & 1.05 \\
\hline 9403.36 & Fe II (71) & 1.08 & 16109.31 & Br13 & 1.05 \\
\hline 9545.974 & $\mathrm{P} 8$ & 1.14 & 16407.19 & Br12 & 1.12 \\
\hline 9590.94 & [Cr II] $(16 \mathrm{~F})$ & 1.08 & 16806.52 & Br11 & 1.10 \\
\hline 9663.588 & Ca II (55) & 1.07 & 17362.11 & Br10 & 1.11 \\
\hline 9997.58 & Fe II & 1.08 & 21655.29 & $\mathrm{Br} \gamma$ & 1.16 \\
\hline
\end{tabular}

${ }^{\text {a }}$ A blend of the higher members of the Paschen series near its limit.

b The line has a P Cyg-type profile with an emission component at $1.07 I_{\text {cont }}$.

temperature $(15000 \mathrm{~K})$, and the estimated interstellar reddening and distance yield the absolute visual luminosity $M_{V} \sim-3.3 \mathrm{mag}$, assuming that MWC 657 is a single star and the circumstellar contribution to its optical brightness is small. According to Strajzhys \& Kurilene (1981), the bolometric correction for such a star is BC $-1.3 \mathrm{mag}$, leading to $\log L_{\mathrm{bol}} / L_{\odot} \leq 3.7$. The uncertainty of this estimate, due to the uncertainties in the distance and the optical brightness, is at least 50 per cent. In addition, a higher temperature would result in a higher luminosity, although this may be partially canceled by a reduction for the possible circumstellar continuum emission.

The spectroscopic data have been obtained at different phases (the OHP 1994 spectrum was taken at phase 0.84 , the OHP 1995 at 0.89 , the DSO 1998 at 0.95 , the SAO 1999 at 0.33 , while the DSO 1999 spectrum was obtained at phase 0.46 ), but mainly close to the photometric minimum. Nevertheless, the observed line profile changes seem to suggest that the periodicity is real. On the other hand, the last spectrum obtained at DSO nearly at the photometric maximum turned out to be almost identical to the first DSO spectrum. This result may indicate that the variations are difficult to detect at this resolution, or that the object's variability is more complicated than simple cyclicity.

\subsection{Modelling}

Since the circumstellar envelope of MWC 657 is most likely non-spherical and very dense, we did not model its
Balmer line profiles, as the modelling of such structures is not yet well developed (e.g. Hummel 2000). The SED of MWC 657 has been modelled by Miroshnichenko et al. (1997). In this section we concentrate on modelling the SED and Balmer line profiles of AS 78.

The near- and far-IR excesses observed in the SED of AS 78 suggest that the object is surrounded by a dusty envelope. In order to model the dusty environments we constructed the SED using our photometry, the MSX, and the IRAS fluxes. The models were calculated with a code by Ivezić et al. (1999), which solves the radiative transfer problem in a spherical dusty envelope. The temperature structure of the envelope is calculated self-consistently taking into account absorption, emission, and scattering of the stellar radiation in the envelope.

The calculations were performed for stellar temperatures $T_{\text {eff }}$ in the range from $16000 \mathrm{~K}$ to $22000 \mathrm{~K}$, which corresponds to B2-B5 spectral types (Strajzhys \& Kurilene 1981). The stellar radiation was modeled with Kurucz (1994) model atmospheres. The dust temperature at the inner envelope's boundary $\left(T_{\text {in }}\right)$ was varied between 1000 and $1500 \mathrm{~K}$. Since no reliable information about the $10-\mu \mathrm{m}$ silicate feature is available, we used the optical properties of two different grain chemical compositions: interstellar dust (Mathis et al. 1977, hereafter MRN) and amorphous carbon (Hanner 1988). The density distribution in the envelope was chosen to be a power-law $r^{-\alpha}$, where $r$ is the distance from the star. The steep decrease of the far-IR fluxes implies a relatively small ratio $\left(Y_{\text {out }}\right)$ of the outer to inner radii and a small optical depth of the envelope $\left(\tau_{V}\right)$; these are two other parameters of the 


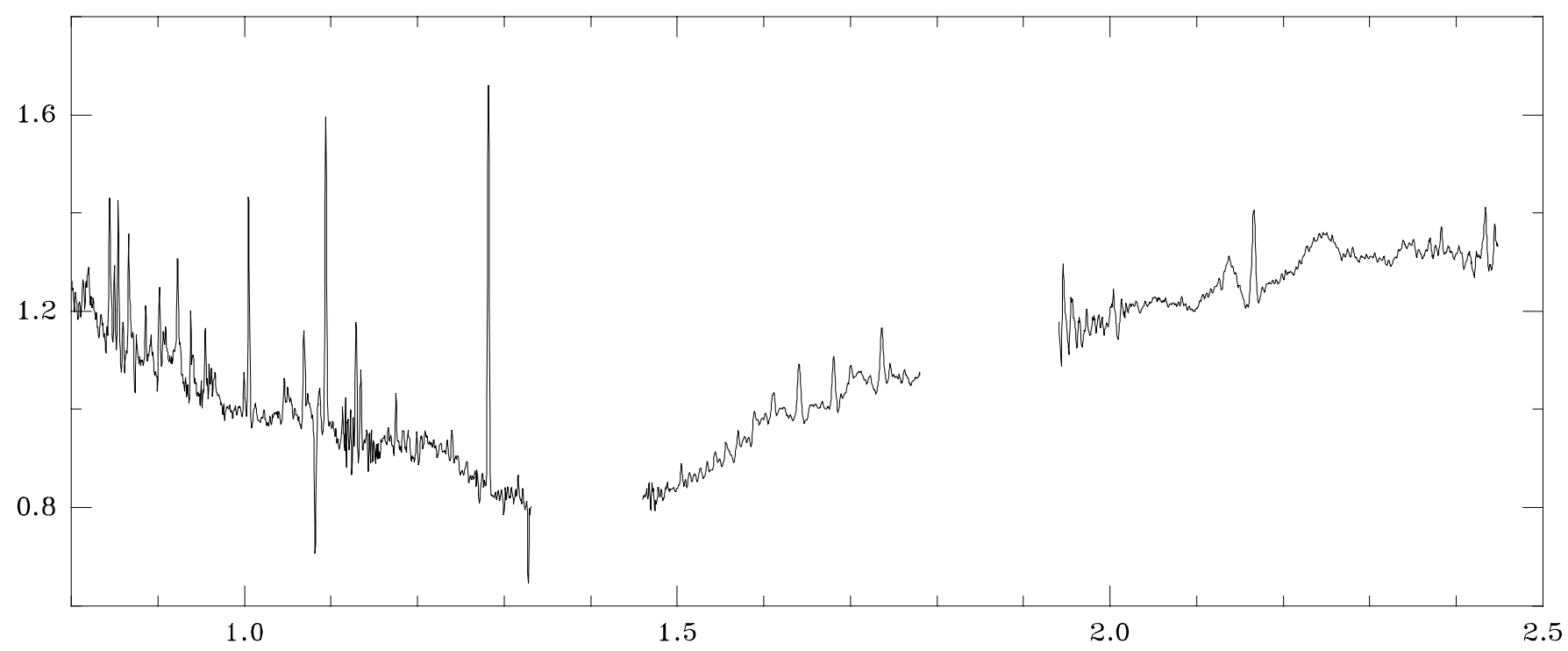

Fig. 9. The near-IR spectrum of AS 78. The wavelengths are given in $\mu \mathrm{m}$, while the flux $F_{\lambda}$ in units of $10^{-16} \mathrm{~W} \mathrm{~cm}^{-1} \mu \mathrm{m}^{-1}$
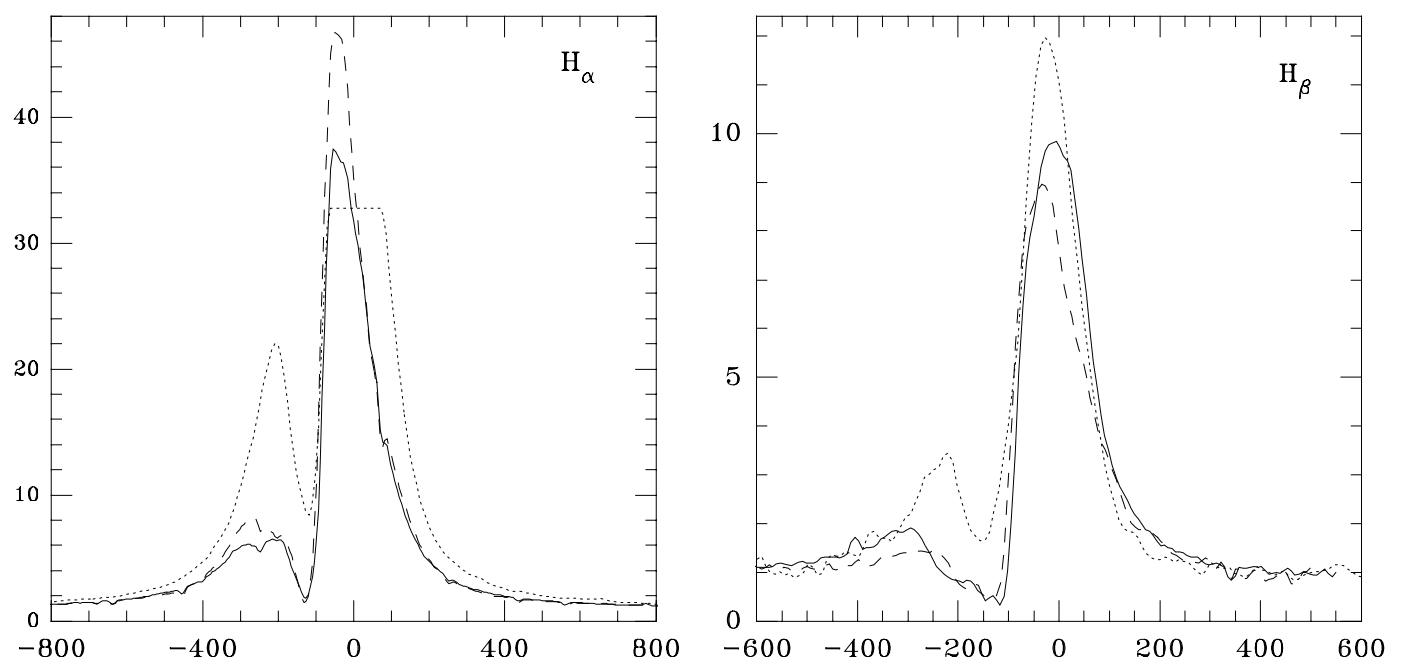

Fig. 10. The $\mathrm{H} \alpha$ (left panel) and $\mathrm{H} \beta$ (right panel) line profile variations in the spectrum of MWC 657 . The OHP 1994 spectrum is shown by a solid line, that of OHP 1995 by a dashed line, while that of SAO 1999 by a dotted line (the redshifted peak of $\mathrm{H} \alpha$ is saturated). Units of the velocities and intensities are the same as in Fig. 7

modelling. Since both the interstellar and circumstellar contributions to the overall extinction are not known a priori, we treated $A_{V}$ as an additional free parameter and derived it by comparing the observed and modelled SED.

The optical part of the SED is dominated by the star's radiation, so that $T_{\text {eff }}$ controls the $A_{V}$. The best fit with $A_{V}=2.86 \mathrm{mag}$, which is in accord with the above independent estimates, is found for $T_{\text {eff }}=17000 \mathrm{~K}, \tau_{V}=0.12$, $Y_{\text {out }}=1000, T_{\text {in }}=1200 \mathrm{~K}$, a power-law density index $\alpha=$ 1.6, and amorphous carbon grains. The theoretical SEDs for the MRN mixture give a much poorer fit. Changes in the stellar surface gravity do not affect the quality of the fits. The observed SED, dereddened with $A_{V}=2.86 \mathrm{mag}$, and the corresponding best fits for both types of dust are presented in Fig. 11 in units of the bolometric flux.

Despite the fact that the spherical envelope fit is close to the observed SED, such a geometry does not solve the near-IR variation problem. It is hard to imagine that the star lost a significant part of its dusty envelope in a few weeks (between 1999 August 28 and September 17). The distance between the star and the dust, which radiates the bulk of $2-\mu \mathrm{m}$ emission, is several hundreds of stellar radii. This requires a dramatic decrease in the star's luminosity or a matter outflow with a speed of about $0.01 \mathrm{c}$ to push the dust outwards. Both these suggestions seem to be unlikely, given the star's long-term stability in the optical region. 
A possible solution of this problem is the suggestion that the dust is distributed non-spherically. Recently Miroshnichenko \& Corporon (1999) showed that a SED, similar to what we see in $\mathrm{AS} 78$, in the region longward of $1 \mu \mathrm{m}$ can be produced by an optically-thick and geometrically-thin disk. Such a disk would have an outer temperature of a few hundred Kelvin and would be much smaller than the spherical envelope producing the same SED, because the disk is much cooler due to its high optical depth. The latter property makes the disk spectrum flat in the $10-\mu \mathrm{m}$ region. However, a weak $10-\mu \mathrm{m}$ emission could exist, because some dust may still be present in the form of a spherical envelope. We might speculate about a suggested disk surrounding a secondary companion, the luminosity of which is not large enough to make a noticeable contribution to the observed radiation of the system. This might explain why we do not see brightness modulation in the optical region. Such a disk's contribution to the radiation in the $J$ and $H$ bands would be rather small, but would become dominant longward of approximately $2 \mu \mathrm{m}$. Its effective radius for the $2 \mu \mathrm{m}$ radiation is a few radii of the secondary, so that it can be occulted easily by the primary. The photometric variations should be smaller at longer wavelengths, where the disk radiates at larger radii. Thus, the binary hypothesis is capable of explaining both the IR excess and the near-IR variations. However, current information about the possible secondary is insufficient to model the SED of such a binary.

In order to put further constraints on the physical characteristics of the star and to estimate the parameters of its wind, we tried to calculate the Balmer line profiles by means of the method described by Pogodin (1986) for spherically symmetric envelopes. The spherical model usage is justified by the P Cyg-type profiles, which can be produced either in a spherical or a geometricallythick disk-like envelope. Some initial ideas about the wind kinematics can be obtained from comparing the object's line profiles with those of well-studied stars, which display similar emission-line spectra. Recently the results of the analysis of the Balmer and He I lines of $\mathrm{P}$ Cyg and HDE 316285 have been published (Najarro et al. 1997 and Hiller et al. 1998, respectively). The line profiles of both stars were well fitted with the same $\beta$-velocity law ( $\beta=$ 2.5 ), with wind terminal velocities of 185 ( $\mathrm{P} \mathrm{Cyg}$ ) and $410 \mathrm{~km} \mathrm{~s}^{-1}$ (HDE 316285), and with the wind density parameters $\left(\dot{M} R_{*}^{-1.5}\right)$ of $4.610^{-8}$ (P Cyg) and $3.710^{-7}$ (HDE 316285) $M_{\odot} \mathrm{yr}^{-1} R_{\odot}^{-1.5}$. Comparison of the $\mathrm{H} \alpha$ profiles of the three objects shows that their emission component cores have almost the same blue and red slope which suggests $\beta=2.5$ for AS 78 (see Fig. 12). The wind terminal velocity of AS 78 is nearly $500 \mathrm{~km} \mathrm{~s}^{-1}$ based on the red border of the absorption feature. The full width at half maximum (FWHM) of the emission component of $\mathrm{AS} 78$ is close to that of $\mathrm{P} \mathrm{Cyg}$, while the absorption component of AS 78 is much wider than those of both $\mathrm{P}$ Cyg and HDE 316285. The latter fact leads to the suggestion that the acceleration in the outer wind regions of AS 78 is significantly smaller than in those of the other two stars and that the $\beta$-velocity law does not work there. The wind density parameter does not seem to be smaller than that of $\mathrm{P}$ Cyg, since both the $\mathrm{H} \alpha$ and $\mathrm{H} \beta$ lines in $\mathrm{AS} 78$ are stronger than those of $\mathrm{P}$ Cyg. This suggestion leads to a rough mass loss rate estimate of $\dot{M} \geq 1.510^{-6} M_{\odot} \mathrm{yr}^{-1}$.

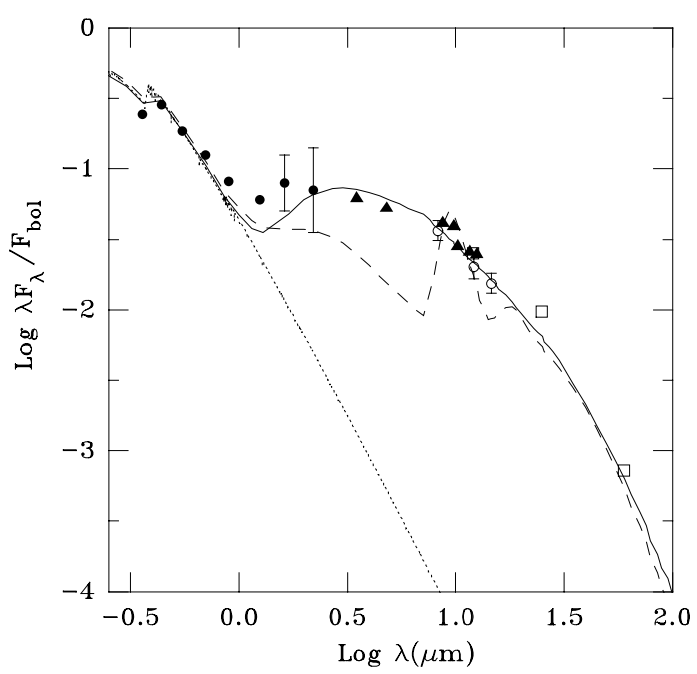

Fig. 11. Fitting the SED of AS 78. The dereddened SED of AS 78 normalized to the bolometric flux is shown by filled circles (averaged Tien-Shan, CST, and Lick data), filled triangles (IRTF data), open circles (MSX data), and open squares (IRAS data). The best fit for MRN dust is shown by a dashed line, and for amorphous carbon dust by a solid line. The SED of a star with $T_{\text {eff }}=17000 \mathrm{~K}$ and $\log g=3.0$ (Kurucz 1994) is shown by a dotted line

Here we try to check the above estimates on the low-resolution line profiles (the SAO 1994 data). The kinematics of the stellar wind was described through the $\beta$ velocity law of Cohen \& Barlow (1977): $w(x)=w_{0}+(1-$ $\left.w_{0}\right)(1-1 / x)^{\beta}$, where $w(x)=v(x) / v_{\infty}, w_{0}=w(x=1)$, $v(x)$ is the velocity at the dimensionless distance $x$ from the stellar center, which is expressed here in stellar radii, and $v_{\infty}$ is the terminal velocity of the stellar wind. Stellar radiation and photospheric line profiles were introduced using Kurucz (1979) model atmospheres. The wind temperature was assumed constant throughout the envelope at a level of $80 \%$ of $T_{\text {eff }}$ (Lamers \& Waters 1984). The photospheric line profiles were broadened by the star's rotation of $70 \mathrm{~km} \mathrm{~s}^{-1}$ (see Sect. 3.2.1). For each model the radial electron scattering optical depth $\left(\tau_{\mathrm{e}}\right)$ was calculated, and the emergent line profiles were corrected for electron scattering using the method by Castor et al. (1970). For comparison with the observations, we convolved the resulting profiles with an instrumental Gaussian profile in order to achieve a resolution of $2 \AA$.

With these assumptions we calculated a grid of models for different $T_{\text {eff }}, \dot{M} R_{*}^{-1.5}, \log g, \beta, w_{0}$, and $v_{\infty}$. 


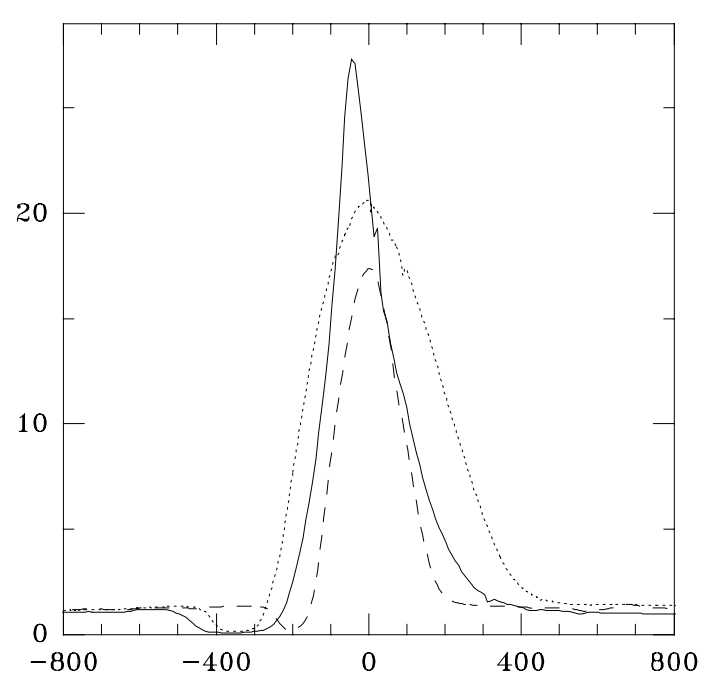

Fig. 12. The $\mathrm{H} \alpha$ line profiles of AS 78 (solid line), P Cyg (dashed line), and HDE 316285 (dotted line). Units of the velocities and intensities are the same as in Fig. 7

The best result was obtained for the following parameter values: $T_{\text {eff }}=17000 \mathrm{~K}, \log g=3.0, v_{\infty}=350 \mathrm{~km} \mathrm{~s}^{-1}$, $w_{0}=0.15, \dot{M} R_{*}^{-1.5}=3.810^{-8} M_{\odot} \mathrm{yr}^{-1} R_{\odot}^{-1.5}, \beta=4.5$, $\tau_{\mathrm{e}}=0.5$. These fits are shown in Fig. 13. The star's radius, calculated from the above bolometric luminosity estimate and $T_{\text {eff }}$, turns out to be $11.5 R_{\odot}$. The wind density parameter and $R_{*}$ give the mass-loss rate estimate $\dot{M}=$ $1.510^{-6} M_{\odot} \mathrm{yr}^{-1}$, which is in good agreement with the lower level derived from the comparison with $\mathrm{P}$ Cyg.

The observed absorption components of the $\mathrm{H} \gamma$ and $\mathrm{H} \delta$ lines are wider than the theoretical ones at the blue side. This can be due to the poor resolution, simplicity of the model, and/or blending with nearby weak absorption lines (e.g., with those of $\mathrm{O}$ II Mult. 18 which are located at $-400-600 \mathrm{~km} \mathrm{~s}^{-1}$ from the center of the $\mathrm{H} \delta$ line). Nevertheless, the agreement between the observations and model results is good especially for the $\mathrm{H} \beta$ line. The models with larger $T_{\text {eff }}$ have shallower absorption components in the $\mathrm{H} \beta-\mathrm{H} \delta$ lines with respect to the observed ones. A similar effect is seen for the models with a larger wind density parameter $\dot{M} R_{*}^{-1.5}$, which leads to a larger $\tau_{\mathrm{e}}$. Smaller values of $w_{0}$ also produce the same effect, while a smaller $\beta$ results in shallower Balmer decrements.

These modelling results show that in 1994 the wind of AS 78 probably had a lower acceleration than in 1999. The difference in the Balmer decrement noticed above and the results of the $\mathrm{H} \alpha$ profile comparison with those of other stars (Sect. 3.2.1) indicate that the wind structure has changed during the period between our observations. Qualitatively these changes can be understood as follows: the wind acceleration has increased, and the denser regions near the star's surface were moved outwards resulting in a wider P Cyg-type absorption component. The massloss rate has most likely increased as well. This scenario requires a characteristic time of nearly a year, since this is roughly the time for the gas to travel all the way through the envelope outer boundary $\left(\sim 100 R_{*}\right)$ at a velocity of $100 \mathrm{~km} \mathrm{~s}^{-1}$. At this point, we will not attempt to model our high-resolution data until new spectra are obtained.

\section{Discussion}

\section{1. $A S 78$}

Now we can take into account all the observed and derived properties of AS 78 in order to discuss its possible evolutionary state. With the fundamental parameters derived above, the star is clearly located above the main sequence. Direct comparison with the evolutionary tracks of Schaller et al. 1992 yields a mass for the star of $M_{*} \sim$ $9 M_{\odot}$ (Fig. 14). The bright emission-line spectrum and the presence of a strong near-IR excess due to hot circumstellar dust make AS 78 similar to B[e] supergiants (Zickgraf et al. 1986). Its position in the HR diagram is close to that of HD 87643, which shows some features of this type of object (e.g., Oudmaijer et al. 1998). However, most B[e] supergiants are much more luminous (see Fig. 14) and more massive. One can argue that these stars might have lost a significant fraction of their masses through the stellar wind. This matter would appear as a nebula surrounding the stars which is seen in HD 87643, but not in AS 78 . Therefore, this interpretation is hardly applicable to the latter.

Let us now explore other options for the nature of AS 78. The location outside the main sequence rules out the possibility that it is a pre-main-sequence Herbig Ae/Be star. The calculations by Palla \& Stahler (1993) and their analysis of the distribution of young intermediate-mass stars in the HR diagram show that they would still be hidden inside their parental clouds if they were as far from the zero-age main-sequence as AS 78 is now. Even if they are visible, they should be surrounded by a large amount of protostellar dust, which produces a significant far-IR excess (e.g., Berrilli et al. 1992). The SED of AS 78 clearly shows the lack of such an excess.

AS 78 may be a post-AGB object because of its intermediate luminosity. According to Blöcker (1995), the parameters we derived ( $L_{\mathrm{bol}}$ and $\left.T_{\text {eff }}\right)$ would correspond to a star with an initial mass $M_{\mathrm{ZAMS}}=4 M_{\odot}$ and a core mass $M_{\mathrm{H}}=0.7 M_{\odot}$ nearly 50 years after the beginning of its post-AGB evolution. Usually these intermediate-mass stars evolve through the post-AGB phase to the planetary nebula phase very quickly. During this evolutionary phase, they are usually heavily obscured by the circumstellar envelope which was ejected during the previous AGB phase. In fact, all type I (massive) and most of the type II (less massive) PNe are known to be strong emitters in the far infrared having the peak of emission between 25 and 

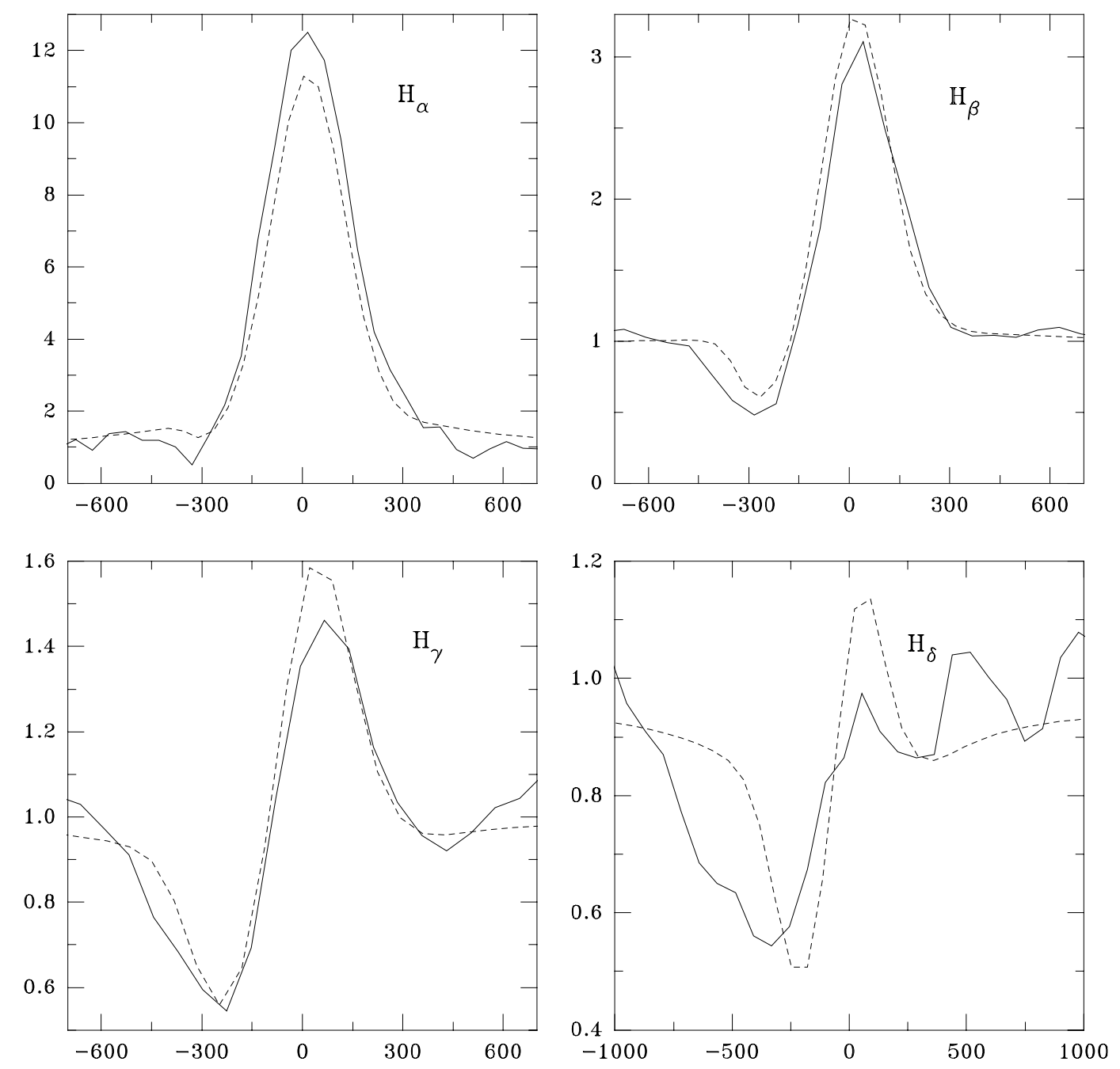

Fig. 13. Best theoretical fits (dashed lines) to the low-resolution Balmer line profiles of AS 78 obtained on 1994 January 21 (solid lines). Units of the velocities and intensities are the same as in Fig. 7

$60 \mu \mathrm{m}$ (e.g. Pottasch et al. 1988). Thus, this possibility seems to be very unlikely for AS 78 .

The presence of a large number of Fe II lines in the spectrum and the shape of the IR excess make AS 78 similar to the group $2 \mathrm{~B}[\mathrm{e}]$ stars (26 objects) selected by Allen \& Swings (1976). The nature and evolutionary state of most of these stars have not yet been determined (e.g. Lamers et al. 1998). However, a few objects from this group (e.g., GG Car, MWC 342, MWC 84, MWC 623) have been suspected to be binaries (see Miroshnichenko 1998 for a recent review). MWC 84 (CI Cam) and MWC 342 (V1972 Cyg) both show strong Balmer and Fe II emission-line spectra as well as IRAS fluxes rapidly decreasing towards longer wavelengths. MWC 342 displays cyclic variations of its optical brightness, while CI Cam was detected as a strong X-ray transient source in 1998. Nevertheless, the presence of their secondary components can be detected only indirectly. This suggests that these components are rather compact. At the same time, all quoted suspected $\mathrm{B}[\mathrm{e}]$ binaries display very strong emission-line spectra, which are usually not observed in single non-supergiant stars.

AS 78 also shows such a strong emission-line spectrum. Moreover, as we speculated in Sect. 3.3, the near-IR variations detected in AS 78 might imply that its secondary is surrounded by a dusty disk, which is sometimes occulted by the primary. Location of the dusty disk around the visible component seems to be unrealistic since the latter's gaseous envelope does not show a noticeable flattening (see Sect. 3.3). That fact we have not found any periodicity in the photometric variations of AS 78 might suggest that the binary separation is large and the corresponding orbital period is comparable with the period of our observations. Thus, the binary hypothesis agrees with the information we derive for AS 78 better than any other considered above, but it still requires additional observations to be proven. 


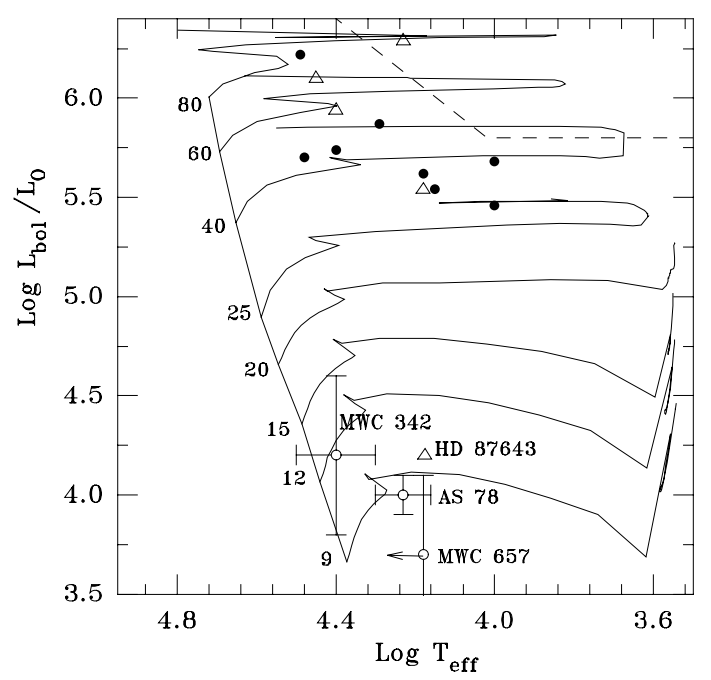

Fig. 14. Hertzsprung-Russell diagram. The zero-age mainsequence and evolutionary tracks from Schaller et al. (1992) for stars of different initial masses (denoted by the numbers in $M_{\odot}$ near corresponding tracks) are shown by solid lines, the Humphreys-Davidson limit by a dashed line. Positions of LBVs and candidate LBVs collected from literature are shown by filled circles, those of some high-luminosity B $[\mathrm{e}]$ supergiants by triangles. The positions of AS 78 and MWC 657 (this work), MWC 342 (Miroshnichenko \& Corporon 1999), and HD 87643 (Zorec 1998) are shown by the open circles

\section{2. $M W C 657$}

Despite the fact that the spectra of MWC 657 and AS 78 are quite similar, it can be seen from the above discussion that their photometric and spectroscopic properties are different. MWC 657 seems to be surrounded by a dense circumstellar gaseous disk, while the emission-line spectrum of AS 78 is more likely due to a mostly spherical stellar wind. The photometric variations of MWC 657 show signs of periodicity, while those of AS 78 are rather gradually changing with time. These differences might imply a different nature and/or evolutionary state for the objects. However, these differences may be due to different contributions of the system components to the overall radiation.

The position of MWC 657 as a single star in the HR diagram is close to that of AS 78 (Fig. 14). Therefore, all the above arguments about the $\mathrm{B}[\mathrm{e}]$ supergiant, pre-mainsequence Herbig Ae/Be star, and post-AGB scenarios that we applied to AS 78 are valid for MWC 657. Moreover, the observed properties of MWC 657 resemble those of MWC 84 and MWC 342, as in the case of AS 78. The spectral line profiles of MWC 342 (which are very similar to those of MWC 657) and CI Cam suggest that they are formed in dense gaseous disks. The shape of the IR excess of MWC 657 (which is almost identical to those of AS 78, MWC 342, and MWC 84) may be understood if it is formed in the circumstellar disk. Due to these similarities, the binary hypothesis seems to be a very attractive explanation of the behaviour of MWC 657 .

Let us assume that the photometric variations are only due to the variable contribution from the secondary component + disk. Then the upper limit of the $V$-band brightness of the visible component is nearly 13.2 mag. This, in combination with the interstellar $E_{B-V}=1.1$ and $D=3.5 \mathrm{kpc}$, gives us its absolute visual magnitude $M_{v} \geq-2.8 \mathrm{mag}$. The presence of the broad He I absorption lines, which most likely belong to the visible component, indicates that it is a moderately rotating B-type star. The width and strength of the He I 5876 and $6678 \AA$ lines in the 1994 OHP spectrum are consistent with a temperature for the star of $15000 \mathrm{~K}$ and $v \sin i \sim 100 \mathrm{~km} \mathrm{~s}^{-1}$ (see Fig. 7), assuming no disk emission contribution to the profiles. The same $\mathrm{BC}=-1.3 \mathrm{mag}$ as in Sect. 3.2.2 gives $M_{\mathrm{bol}} \geq-4.1 \mathrm{mag}, \log L_{\mathrm{bol}} / L_{\odot} \leq 3.5$, and a radius for the star of $R_{*} \leq 10 R_{\odot}$. Assuming the total mass of the system is $10 M_{\odot}$, the orbital period of 86 days gives a separation between the components of $\sim 10^{13} \mathrm{~cm}$. This value is an order of magnitude larger than the deduced radius of the brighter component. Thus, the brighter component does not fill its Roche lobe and is the presumed mass gainer.

Since no spectral lines of the fainter (secondary) component are seen, it is most likely that the disk fills the Roche lobe of this component and is the source of mass transfer in the system. Such a scenario has been developed for massive X-ray binaries by Harmanec (1985), one of which may well be MWC 657. If this is the case, the fainter component of MWC 657 is probably a compact helium star, which means that the system is rather evolved. To get more information about both possible components, one needs high-resolution spectroscopy of MWC 657 at the minimum and maximum brightness phases.

\section{Conclusions}

We have obtained and analysed photometric and spectroscopic data for two peculiar emission-line stars AS 78 and MWC 657 . Both objects were found to display noticeable photometric variations in the optical region with an amplitude of about 0.3 and $0.6 \mathrm{mag}$, respectively. Highresolution spectra were obtained for both objects for the first time. We find that they both lack photospheric lines and exhibit variations in the emission line profiles. The IR excesses are also similar in AS 78 and MWC 657 indicating a lack of far-IR radiation. Both objects were identified in the recent mid-IR galactic plane survey of the MSX satellite with fluxes comparable to those of IRAS.

A near-IR excess is detected for AS 78 for the first time. A gradual decrease of its $U-B$ and $B-V$ and simultaneous increase of the $V-R$ and $V-I$ colour-indices was observed during more than 6 years of observations. Variations in the near-IR radiation on the order of $1 \mathrm{mag}$ were found with a time scale of weeks. The radial velocity 
measurements in this spectrum suggest that the distance towards AS 78 is $2.9 \mathrm{kpc}$, based on the galactic rotation curve. The consideration of different hypotheses about the nature and evolutionary state of AS 78 enabled us to discard those of a B[e] supergiant, Herbig Ae/Be star, and post-AGB object. The observed variations of the near-IR flux are more consistent with the binary hypothesis. If this is the case, then the primary is responsible for the bulk of the optical radiation and had $T_{\text {eff }} \sim 17000 \mathrm{~K}$ at the epoch of our first spectroscopic observations (January 1994); $T_{\text {eff }}$ and/or the stellar wind strength appears to be increasing with time.

The high-resolution spectrum obtained in 1999 indicates changes in the wind density structure in comparison with the 1994 data. The secondary must be a less luminous star which is probably surrounded by an optically-thick and geometrically-thin gaseous and dusty disk. Although we currently do not have sufficient evidence for the object's binarity, the observed strong emission-line spectrum of AS 78 is rather unusual for such a relatively low-mass star.

Cyclical photometric variations with a period of 86 days were suspected for MWC 657. The optical colourindices tend to become bluer as the star brightens in the $V$-band. The $K$-band brightness tends to increase significantly towards phase 0.5 , which corresponds to a weak secondary minimum. The double-peaked Balmer emission lines probably show phase dependent $V / R$ changes. We suggest that all these phenomena might be due to the orbital motion in a close binary system, where the gaseous disk around the invisible star fills its Roche lobe and pours matter onto the visible component. The latter is probably a B-type giant star. Such a system is rather unusual, but not completely unexpected.

The observations obtained so far do not allow us to derive the objects' parameters with high accuracy. Additional observations including optical and IR photometry and high-resolution spectroscopy are required for both stars. Nevertheless, this study has revealed a number of their important properties, and our results allow us to create an observing strategy for both. In particular, frequent near-IR observations seem to be the most important to get more information about the IR emitting regions and possible secondary components.

Acknowledgements. We are grateful to B. Kallemov and T.M. Pogrosheva for their help in the photometric observations, N.V. Borisov for his help in the spectroscopic observations with the TV scanner, N.E. Kurochkin for his work with the Moscow plate archive, and P. Corporon for providing us with his high-resolution spectra of MWC 657 . A.M. and K.S.B. acknowledge support from NASA grant NAG5-8054 and thank the IRTF staff for their assistance during the observations. Karen Bjorkman is a Cottrell Scholar of the Research Corporation, and gratefully acknowledges their support. P.G.-L. acknowledges support from grant PB97-1435-C02-02 from the Spanish Dirección General de Enseñanza Superior e Investigación Científica (DGESIC). V.K. acknowledges support from grant 99-02-18339 of the Russian Foundation for Basic Research. R.J.R., D.K.L., and S.M. were supported by the Independent Research and Development program at The Aerospace Corporation. This research has made use of the SIMBAD database operated at CDS, Strasbourg, France.

\section{References}

Allen D.A., Swings J.P., 1976, A\&A 47, 293

Arribas S., Martínez-Roger C., 1987, A\&AS 70, 303

Balega I.I., Vereschagina R.G., Markelov S.V., et al., 1979, Izvestia Spec. Astroph. Obs. 11, 248

Bergner Yu.K., Bondarenko S.L., Miroshnichenko A.S., et al., 1988, Izvestia Glavn. Astron. Obs. v Pulkove 205, 142

Berrilli F., Corciullo G., Ingrosso G., et al., 1992, ApJ 398, 154 Blöcker T., 1995, A\&A 299, 755

Castor J.I., Smith D., van Blerkom D., 1970, ApJ 159, 1119

Cohen L., Barlow M.J., 1977, ApJ 213, 737

Coluzzi R., 1993, Bull. Inf. CDS 43, 7

Corporon P., Lagrange A.-M., 1999, A\&AS 136, 429

Divan L., Zorec J., 1982, in Be stars, Jaschek M., Groth H.-G. (eds.), Proc. IAU Symp. 98, 61

Dong Y.S., Hu J.Y., 1991, Chin. A\&A 15, 275

Dubath P., Mayor M., Burki G., 1988, A\&A 205, 77

Egan M.P., et al., 1999, The Midcourse Space Experiment Point Source Catalog Version 1.2, Explanatory Guide, AFRL-VS-TR-1999-1522

Hammersley P.L., Jourdain de Muizon M., Kessler M.F., et al., 1998, A\&AS 128, 207

Hanner M.S., 1988, Infrared Observations of Comets Halley and Wilson and Properties of the Grains (NASA89-13330), p. 22

Harmanec P., 1985, Bull. Astr. Inst. Czech. 36, 237

Harmanec P., 2000, in The Be Phenomenon in Early-Type Stars, Proc. IAU Coll. 175, Smith M.A., Hendrichs H.F., Fabregat J. (eds.), ASP Conf. Ser. (in press)

Haug U., 1970, A\&AS 1, 35

Herbig G.H., 1993, ApJ 407, 142

Hillier D., Crowther P., Najarro F., Fullerton A.W., 1998, A\&A 340,483

Hoffleit D., Jaschek C., 1982, The Bright Star Catalog. New Haven, Connecticut: Yale University Observatory

Hubeny I., Lanz T., Jeffery C.S., 1995, Synspec - A User's Guide, Version 36

Hummel W., 2000, in The Be Phenomenon in Early-Type Stars, Proc. IAU Coll. 175, Smith M.A., Hendrichs H.F., Fabregat J. (eds.), ASP Conf. Ser. (in press)

Humphreys R.M., 1978, ApJS 38, 309

Humphreys R.M., Lamers H.J.G.L.M., Hoekzema N., Cassatella A., 1989, A\&A 218, L17

Ivezić Ž., Nenkova M., Elitzur M., 1999, User Manual for DUSTY, Univ. of Kentucky Internal report, accessible at http://www.pa.uky.edu/ moshe/dusty

Kuratov K.S., Miroshnichenko A.S., Bergner Yu.K., 1998, in $\mathrm{B}[\mathrm{e}]$ stars, Jaschek C., Hubert A.M. (eds.). Kluwer Academic Publ., p. 165

Koornneef J., 1983, A\&A 128, 84

Kurucz R.L., 1979, ApJS 40, 1 
Kurucz R.L., 1994, Smithsonian Astrophys. Obs., CD-ROM, No. 19

Lamers H.J.G.L.M., Waters L.B.F.M., 1984, A\&A 136, 37

Lamers H.J.G.L.M., Zickgraf F.-J., de Winter D., Houziaux L., Zorec J., 1998, A\&A 340, 117

Mathis J.S., Rumpl, W., Nordsieck K.H., 1977, ApJ 217, 425

Merrill P.W., Burwell C.G., 1933, ApJ 78, 87

Merrill P.W., Burwell C.G., 1950, ApJ 112, 72

Miller W.C., Merrill P.W., 1951, ApJ 113, 624

Miroshnichenko A.S., Kuratov K.S., Ivezić Ž., Elitzur M., 1997, IBVS, 4506

Miroshnichenko A.S., 1998, in B[e] stars, Jaschek C., Hubert A.M. (eds.). Kluwer Academic Publ., p. 145

Miroshnichenko A.S., Frémat I., Houziaux L., et al., 1998, A\&AS 131, 469

Miroshnichenko A.S., Chentsov E.L., Klochkova V.G., 1999, in Variable and Non-Spherical Winds in Hot Luminous Stars, Proc. IAU Coll. 169, Wolf B., Fullerton A., Stahl O. (eds.). Kluwer Acad. Publ., p. 272

Miroshnichenko A.S., Corporon P., Sheikina T.A., 1999, BAAS 31,845

Miroshnichenko A.S., Corporon P., 1999, A\&A 349, 126

Munch G., 1957, ApJ 125, 42

Najarro F., Hillier D., Stahl O., 1997, A\&A 326, 1117

Oudmaijer R.D., Proga D., Drew J.A., de Winter D., 1998, MNRAS 300, 170

Panchuk V.E., Najdenov I.D., Klochkova V.G., et al., 1998, Bull. Spec. Astrophys. Obs. 44, 127

Palla F., Stahler S.W., 1993, ApJ 418, 414

Pogodin M.A., 1986, Afz 24, 491

Pottasch S.R., Olling R., Bignell C., Zijlstra A.A., 1988, A\&A 205, 248

Rubin V.C., 1965, ApJ 142, 934

Rudy R.J., Erwin P., Rossano G.S., Puetter R.C., 1991, ApJ 383, 344

Rudy R.J., Puetter R.C., Mazuk S., 1999, AJ 118, 666

Scargle J.D., 1982, ApJ 263, 835
Schaller G., Schaerer D., Meynet G., Maeder A., 1992, A\&AS 96, 269

Sheikina T.A., Miroshnichenko A.S., 1998, in B[e] stars, Jaschek C., Hubert A.M. (eds.). Kluwer Academic Publ., p. 135

Stock J., et al., 1960, Luminous Stars in the Northern Milky Way, Part II

Strajzhys V., 1977, Multicolour Photometry of Stars, Vilnius, Mokslas

Strajzhys V., Kurilene G., 1981, Ap\&SS 80, 353

Wackerling L.R., 1970, Mem. RAS 73, 153

Waters L.B.F.M., Coté J., Lamers H.J.G.L.M., 1987, A\&A 185, 206

Zickgraf F.-J., Wolf B., Stahl O., et al., 1986, A\&A 163, 119

Zorec J., 1998, in B[e] stars, Jaschek C., Hubert A.M. (eds.). Kluwer Academic Publ., p. 27

\section{List of objects}

"AS 78" on page 5

"MWC 657" on page 5

"HR 1276 " on page 6

"HR 1457" on page 9

"HR 1641" on page 9

"HR 2077" on page 9

"BD $+55^{\circ} 837$ " on page 16

"P Cyg" on page 19

"HDE 316285" on page 19

"HD 87643" on page 20

"GG Car" on page 21

"MWC 342" on page 21

"MWC 84" on page 21

"MWC 623 " on page 21 